\title{
Scheduling and Mapping in an Incremental Design Methodology for Distributed Real-Time Embedded Systems
}

\author{
Paul Pop, Petru Eles, Zebo Peng, Senior Member, IEEE, and Traian Pop
}

\begin{abstract}
In this paper, we present an approach to mapping and scheduling of distributed embedded systems for hard real-time applications, aiming at a minimization of the system modification cost. We consider an incremental design process that starts from an already existing system running a set of applications. We are interested in implementing new functionality such that the timing requirements are fulfilled and the following two requirements are also satisfied: 1) the already running applications are disturbed as little as possible and 2) there is a good chance that later, new functionality can easily be added to the resulted system. Thus, we propose a heuristic that finds the set of already running applications which have to be remapped and rescheduled at the same time with mapping and scheduling the new application, such that the disturbance on the running system (expressed as the total cost implied by the modifications) is minimized. Once this set of applications has been determined, we outline a mapping and scheduling algorithm aimed at fulfilling the requirements stated above. The approaches have been evaluated based on extensive experiments using a large number of generated benchmarks as well as a real-life example.
\end{abstract}

Index Terms-Distributed embedded systems, incremental design, process mapping, process scheduling, real-time systems.

\section{INTRODUCTION}

C OMPLEX embedded systems with multiple processing elements are becoming common in various application areas from telecommunications, automotive electronics, robotics, industrial control, to medical equipment and multimedia. Such systems have to fulfill strong requirements in terms of performance and cost efficiency. There are several complex design steps to be considered during the development of such a system: the underlying architecture has to be allocated (which implies the allocation of components like processors, memories, and buses, together with the decision on a certain interconnection topology), tasks and communication channels have to be mapped on the architecture, and all the activities in the system have to be scheduled. The design process usually implies an iterative execution of these steps until a solution is found such that the resulted system satisfies certain design constraints [10], [12], [24], [25], [39], [42].

Several notable results have been reported, aimed at supporting the designer with methodologies and tools during the hardware/software cosynthesis of embedded systems. Initially, researchers have considered architectures consisting of a single

Manuscript received December 18, 2002; revised September 29, 2003.

The authors are with the Department of Computer and Information Science, Linköping University, Linköping SE-581 83 Sweden (e-mail: paupo@ida.liu.se, petel@ida.liu.se,zebpe@ida.liu.se,trapo@ida.liu.se).

Digital Object Identifier 10.1109/TVLSI.2004.831467 programmable processor and an ASIC. Their goal was to partition the application between the hardware and software domain, such that performance constraints are satisfied while the total hardware cost is kept at a minimum [7], [9], [11], [15]. Currently, similar architectures are becoming increasingly interesting, with the ASIC replaced by a dynamically reconfigurable hardware coprocessor [21].

As a result of fast technological development and of an increasing demand for reliable embedded systems with highly complex functionality, new, more sophisticated architectures, consisting of a large number of interconnected programmable components and ASICs, are now widely used. Such complex systems can be integrated on a single chip (systems on chip) or can be physically distributed over a smaller or wider area (distributed embedded systems). One of the first attempts to address the problems of allocation, mapping, and scheduling in the context of such a complex architecture has been published in [33]. The approach is based on a mixed integer linear programming (MILP) formulation and has the disadvantage of the huge complexity inherent to solving such a model. Therefore, alternative problem formulations and solutions based on efficient heuristics have been proposed [1], [2], [5], [20], [40], [41].

Although much of the above work is dedicated to specific aspects of distributed systems, researchers have often ignored or very much simplified issues concerning the communication infrastructure. One notable exception is [37], in which system synthesis is discussed in the context of a distributed architecture based on arbitrated buses. Many efforts dedicated to communication synthesis have concentrated on the synthesis support for the communication infrastructure but without considering hard real-time constraints and system level scheduling aspects [16], [26], [27].

Another characteristic of research efforts concerning the codesign of embedded systems is that authors concentrate on the design, from scratch, of a new system optimized for a particular application. For many application areas, however, such a situation is extremely uncommon and only rarely appears in design practice. It is much more likely that one has to start from an already existing system running a certain application and the design problem is to implement new functionality on this system. In such a context it is very important to operate none, or as few as possible, modifications to the already running application. The main reason for this is to avoid unnecessarily large design and testing times. Performing modifications on the (potentially large) existing application increases design time and even more testing time (instead of only testing the 
newly implemented functionality, the old application, or at least a part of it, has also to be retested). However, this is not the only aspect to be considered. Such an incremental design process, in which a design is periodically upgraded with new features, is going through several iterations. Therefore, after new functionality has been implemented, the resulting system has to be structured such that additional functionality, later to be mapped, can easily be accommodated [31], [32].

In a recent paper [14], Haubelt et al. consider the requirement of flexibility as a parameter during design space exploration. However, their goal is not incremental design, but the generation of an architecture which, at an acceptable cost, is able to implement different applications or variants of a certain application.

In this paper, we use a nonpreemptive static-cyclic scheduling policy for processes and messages. Using such a scheduling policy is strongly recommended for many types of applications, e.g., hard real-time safety critical applications [17]. In [29] we have discussed the implications of an incremental design process in the context of a fixed-priority preemptive scheduling policy.

\section{A. Contributions}

The contribution of the present paper is twofold.

1) First, we consider mapping and scheduling for hard realtime embedded systems in the context of a realistic communication model based on a time-division multiple-access (TDMA) protocol as recommended for applications in areas like, for example, automotive electronics [18]. We accurately take into consideration overheads due to communication and consider, during the mapping and scheduling process, the particular requirements of the communication protocol.

2) As our main contribution, we have considered, for the first time to our knowledge, the design of distributed embedded systems in the context of an incremental design process as outlined above. This implies that we perform mapping and scheduling of new functionality on a given distributed embedded system, so that certain design constraints are satisfied, and in addition: 1) the already running applications are disturbed as little as possible and 2) there is a good chance that later, new functionality can easily be mapped on the resulted system.

We propose a new heuristic, together with the corresponding design criteria, which finds the set of old applications that have to be remapped and rescheduled at the same time with mapping and scheduling the new application, such that the disturbance on the running system (expressed as the total cost implied by the modifications) is minimized. Once this set of applications has been determined, mapping and scheduling is performed according to the requirements stated above.

Supporting such a design process is of critical importance for current and future industrial practice, as the time interval between successive generations of a product is continuously decreasing, while the complexity due to increased sophistication of new functionality is growing rapidly. The goal of reducing the overall cost of successive product generations has been one

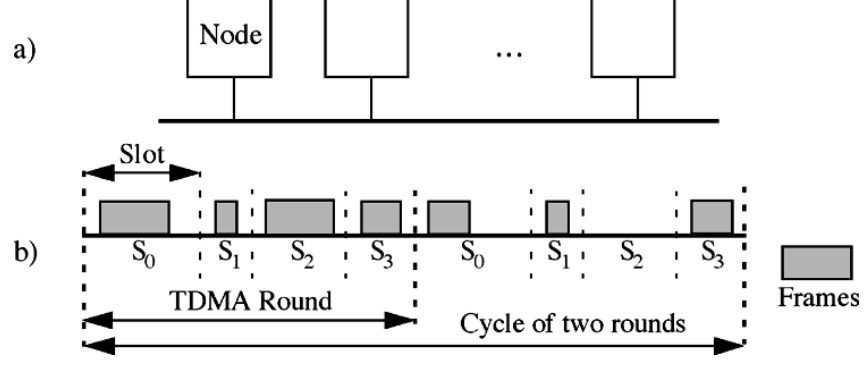

Fig. 1. System architecture.

of the main motors behind the currently very popular concept of platform-based design [19], [23]. Although in this paper we are not explicitly dealing with platform-based systems, most of the results are also valid in the context of this design paradigm.

This paper is organized as follows. Section II presents preliminary discussions concerning the system architecture we consider and our abstract system representation. In Section III, we formulate the problem we are going to solve. Section IV introduces our approach to quantitatively characterize certain features of both currently running and future applications. In Section $\mathrm{V}$, we introduce the metrics we have defined in order to capture the quality of a given design alternative, and based on these metrics we give an exact problem formulation. Our mapping and scheduling strategy is described in Section VI, and the experimental results are presented in Section VII. Finally, Section VIII presents our conclusions.

\section{PRELIMINARIES}

\section{A. System Architecture}

1) Hardware Architecture: We consider architectures consisting of nodes connected by a broadcast communication channel [Fig. 1(a)]. Every node consists of a TTP controller, processor, memory, and an I/O interface to sensors and actuators.

Communication between nodes is based on the TTP [18]. TTP was designed for distributed real-time applications that require predictability and reliability (e.g., drive-by-wire). It integrates all the services necessary for fault-tolerant real-time systems.

The communication channel is a broadcast channel, so a message sent by a node is received by all the other nodes. The bus access scheme is TDMA [Fig. 1(b)]. Each node $N_{i}$ can transmit only during a predetermined time interval, the so called TDMA slot $S_{i}$. In such a slot, a node can send several messages packed in a frame. A sequence of slots corresponding to all the nodes in the architecture is called a TDMA round. A node can have only one slot in a TDMA round. Several TDMA rounds can be combined together in a cycle that is repeated periodically. The sequence and length of the slots are the same for all TDMA rounds. However, the length and contents of the frames may differ.

Every node has a TTP controller that implements the protocol services and runs independently of the node's CPU. Communication with the CPU is performed through a shared memory which is the message base interface (MBI) in Fig. 2. The TDMA access scheme is imposed by a so called message descriptor list 


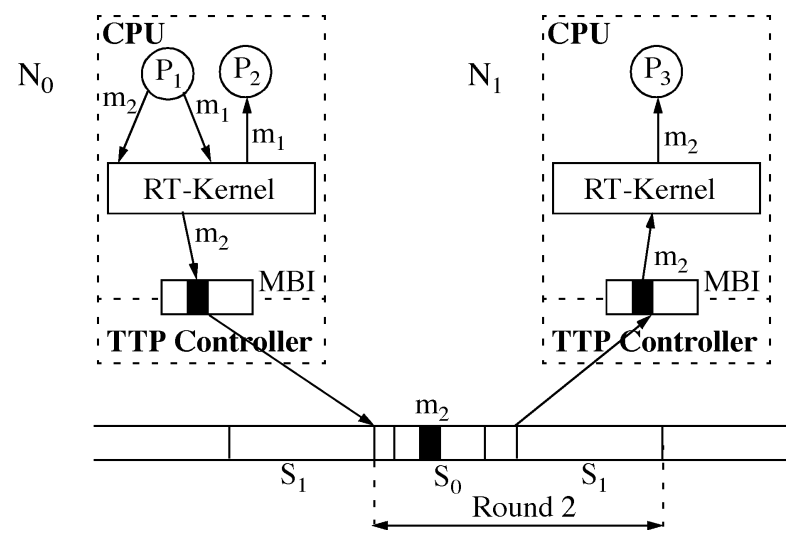

Fig. 2. Message passing mechanism.

(MEDL) that is located in every TTP controller. The MEDL serves as a schedule table for the TTP controller which has to know when to send or receive a frame to or from the communication channel. The TTP controller provides each CPU with a timer interrupt based on a local clock synchronized with the local clocks of the other nodes. Clock synchronization is done by comparing the a priori known time of arrival of a frame with the observed arrival time. Thus, TTP provides a global time-base of known precision, without any overhead on the communication.

2) Software Architecture: We have designed a software architecture which runs on the CPU in each node, which has a realtime kernel as its main component. Each kernel has a schedule table that contains all the information needed to take decisions on activation of processes and transmission of messages, at the predetermined time moments.

The message passing mechanism is illustrated in Fig. 2, where we have three processes, $P_{1}$ to $P_{3} . P_{1}$ and $P_{2}$ are mapped to node $N_{0}$ that transmits in slot $S_{0}$, and $P_{3}$ is mapped to node $N_{1}$ that transmits in slot $S_{1}$. Message $m_{1}$ is transmitted between $P_{1}$ and $P_{2}$ that are on the same node, while message $m_{2}$ is transmitted from $P_{1}$ to $P_{3}$ between the two nodes. We consider that each process has its own memory locations for the messages it sends or receives and that the addresses of the memory locations are known to the kernel through the schedule table.

$P_{1}$ is activated according to the schedule table, and when it finishes it calls the send kernel function in order to send $m_{1}$, and then $m_{2}$. Based on the schedule table, the kernel copies $m_{1}$ from the corresponding memory location in $P_{1}$ to the memory location in $P_{2}$. When $P_{2}$ will be activated it finds the message in the right location. According to our scheduling policy, whenever a receiving process needs a message, the message is already placed in the corresponding memory location. Thus, there is no overhead on the receiving side for messages exchanged on the same node.

Message $m_{2}$ has to be sent from node $N_{0}$ to node $N_{1}$. At a certain time, known from the schedule table, the kernel transfers $m_{2}$ to the TTP controller by packaging it into a frame in the MBI. Later on, the TTP controller knows from its MEDL when it has to take the frame from the MBI, in order to broadcast it on the bus. In our example, the timing information in the schedule table of the kernel and the MEDL is determined in such a way that the broadcasting of the frame is done in the slot $S_{0}$ of Round 2. The TTP controller of node $N_{1}$ knows from its MEDL that it has to read a frame from slot $S_{0}$ of Round 2 and to transfer it into the MBI. The kernel in node $N_{1}$ will read the message $m_{2}$ from the MBI. When $P_{3}$ will be activated based on the local schedule table of node $N_{1}$, it will already have $m_{2}$ in its right memory location.

In [30] we presented a detailed discussion concerning the overheads due to the kernel and to every system call. We also presented formulas for derivation of the worst-case execution delay of a process, taking into account the overhead of the timer interrupt, the worst-case overhead of the process activation and message passing functions.

\section{B. Abstract Representation}

As the basis for abstract modeling we use a directed, acyclic, polar graph $G(V, E)$, called process graph (Fig. 3). Each node $P_{i} \in V$ represents a process. A process is a sequence of computations (corresponding to several building blocks in a programming language), which starts when all its inputs are available and it issues its outputs when it terminates. As mentioned in the introduction, we consider safety-critical applications where not meeting a timing constraint could potentially lead to catastrophic consequences. Hence, each process $P_{i}$ is characterized by a worst-case execution time $C_{i}$. Estimation of the worst-case execution time for a given process has been extensively discussed in the literature [34]. Moreover, we consider a nonpreemptive execution environment. Hence, once activated, a process executes until it completes.

An edge in the process graph, $e_{i j} \in E$ from $P_{i}$ to $P_{j}$ indicates that the output of $P_{i}$ is the input of $P_{j}$.

Each process graph $G$ is characterized by its period $T_{G}$ and its deadline $D_{G} \leq T_{G}$. Deadlines can also be placed locally on processes. Release times of some processes as well as multiple deadlines can be easily modeled by inserting dummy nodes between certain processes and the source or the sink node respectively. These dummy nodes represent processes with a certain execution time but that are not allocated to any processing element.

A process graph is polar, which means that there are two nodes, called source and sink, that conventionally represent the first and last process. If needed, these nodes are introduced as dummy processes so that all other nodes in the graph are successors of the source and predecessors of the sink, respectively.

As shown in Fig. 3, an application $\Gamma$ is modeled as a set of process graphs $G_{i} \in \Gamma$. The whole functionality of the system is represented as a set of applications.

According to our representation model, all processes interacting with each other through time critical messages belong to the same process graph. If processes have different periods, this is solved by generating several instances of processes and building a process graph which corresponds to a set of processes that occur within a time period equal to the least common multiple of the periods of the involved processes. Potential communication between processes in different applications is not part of the model. Technically, such a communication is implemented by the kernels based on asynchronous nonblocking send 


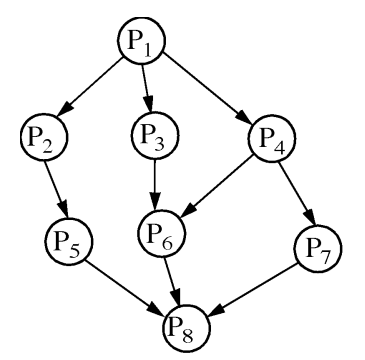

$\underbrace{\text { Process graph } G_{1}}_{\text {Application } \Gamma_{1}} G_{2}$

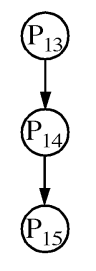

(P.)
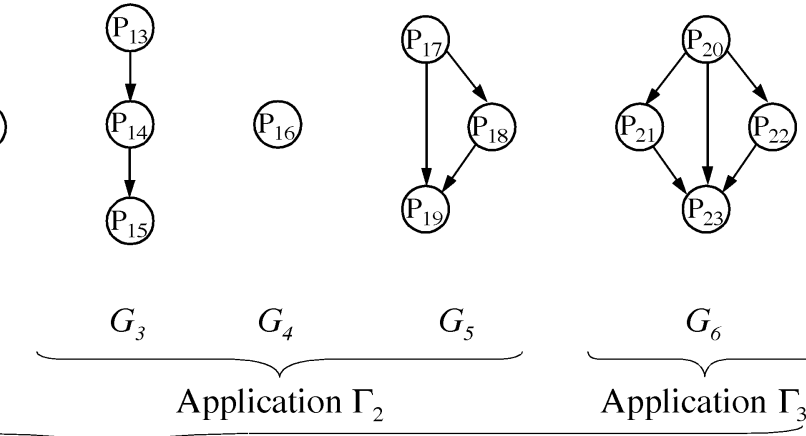

$\underbrace{G_{6}}_{\text {Application } \Gamma_{3}}$

System functionality

Fig. 3. Abstract representation.

and receive primitives. Such messages are considered noncritical and are not affected by real-time constraints. They will use bus slots that have not been assigned to time-critical messages. Therefore, communications of this nature will not be addressed in this paper.

\section{Application Mapping and Scheduling}

Considering a system architecture like the one presented in Section II-A, the mapping of a process graph $G(V, E)$ is given by a function $M: V \rightarrow P E$, where $P E=\left\{N_{1}, N_{2}, \ldots, N_{\text {npe }}\right\}$ is the set of nodes (processing elements). For a process $P_{i} \in V, M\left(P_{i}\right)$ is the node to which $P_{i}$ is assigned for execution. Each process $P_{i}$ can potentially be mapped on several nodes. Let $\mathcal{N}_{P_{i}} \subseteq P E$ be the set of nodes to which $P_{i}$ can potentially be mapped. For each $N_{i} \in \mathcal{N}_{P_{i}}$, we know the worst-case execution time $t_{P_{i}}^{N_{i}}$ of process $P_{i}$, when executed on $N_{i}$. Messages transmitted between processes mapped on different nodes are communicated through the bus, in a slot corresponding to the sending node. The maximum number of bits transferred in such a message is also known.

In order to implement an application, represented as a set of process graphs, the designer has to map the processes to the system nodes and to derive a static cyclic schedule such that all deadlines are satisfied. We first illustrate some of the problems related to mapping and scheduling, in the context of a system based on a TDMA communication protocol, before going on to explore further aspects specific to an incremental design approach.

Let us consider the example in Fig. 4 where we want to map an application consisting of four processes $P_{1}$ to $P_{4}$, with a period and deadline of $50 \mathrm{~ms}$. The architecture is composed of three nodes that communicate according to a TDMA protocol, such that $N_{i}$ transmits in slot $S_{i}$. For this example, we suppose that there is no other previous application running on the system. According to the specification, processes $P_{1}$ and $P_{3}$ are constrained to node $N_{1}$, while $P_{2}$ and $P_{4}$ can be mapped on nodes $N_{2}$ or $N_{3}$, but not $N_{1}$. The worst-case execution times of processes on each potential node and the sequence and size of TDMA slots, are presented in Fig. 4. In order to keep the example simple, we suppose that the message sizes are such that each message fits into one TDMA slot.

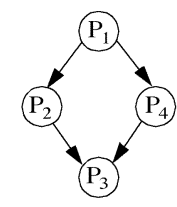

$\mathrm{T}=\mathrm{D}=50 \mathrm{~ms}$

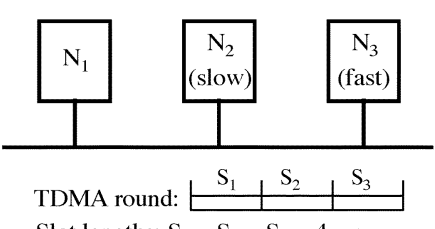

Slot lengths: $S_{1}=S_{2}=S_{3}=4 \mathrm{~ms}$
Process execution times
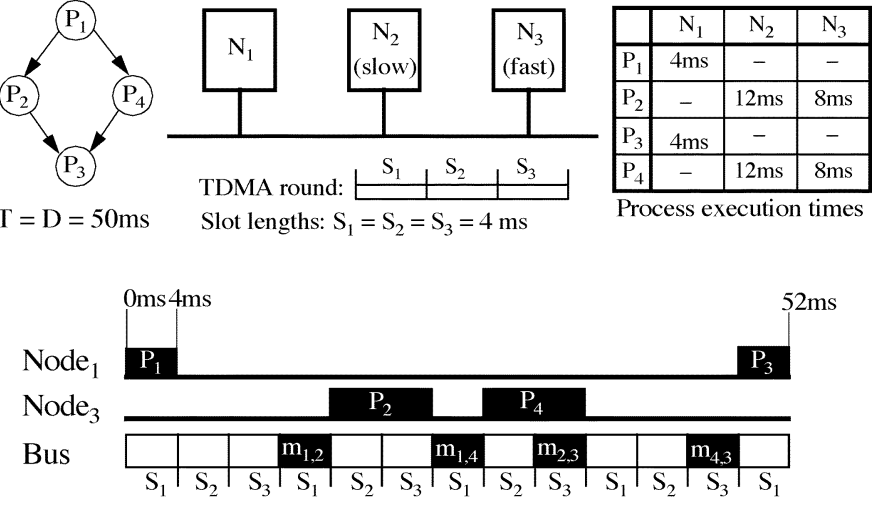

a) Processes $P_{2}$ and $P_{4}$ are mapped on the fast node

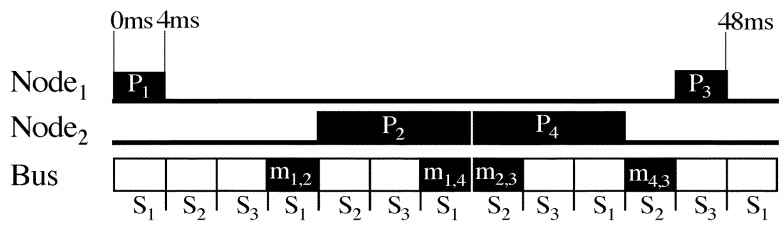

b) Processes $\mathrm{P}_{2}$ and $\mathrm{P}_{4}$ are mapped on the slow node

Fig. 4. Mapping and scheduling example.

We consider two alternative mappings. If we map $P_{2}$ and $P_{4}$ on the faster processor $N_{3}$, the resulting schedule length [Fig. 4(a)] will be $52 \mathrm{~ms}$ which does not meet the deadline. However, if we map $P_{2}$ and $P_{4}$ on the slower processor $N_{2}$, the schedule length [Fig. 4(b)] is $48 \mathrm{~ms}$, which meets the deadline. Note, that the total traffic on the bus is the same for both mappings and the initial processor load is 0 on both $N_{2}$ and $N_{3}$. This result has its explanation in the impact of the communication protocol. $P_{3}$ cannot start before receiving messages $m_{2,3}$ and $m_{4,3}$. However, slot $S_{2}$ corresponding to node $N_{2}$ precedes in the TDMA round slot ${ }_{S} 3$ on which node $N_{3}$ communicates. Thus, the messages which $P_{3}$ needs are available sooner in the case $P_{2}$ and $P_{4}$ are, counter intuitively, mapped on the slower node.

But finding a valid schedule is not enough if we are to support an incremental design process as discussed in the introduction. In this case, starting from a valid design we have to improve the mapping and scheduling so that not only the design constraints 


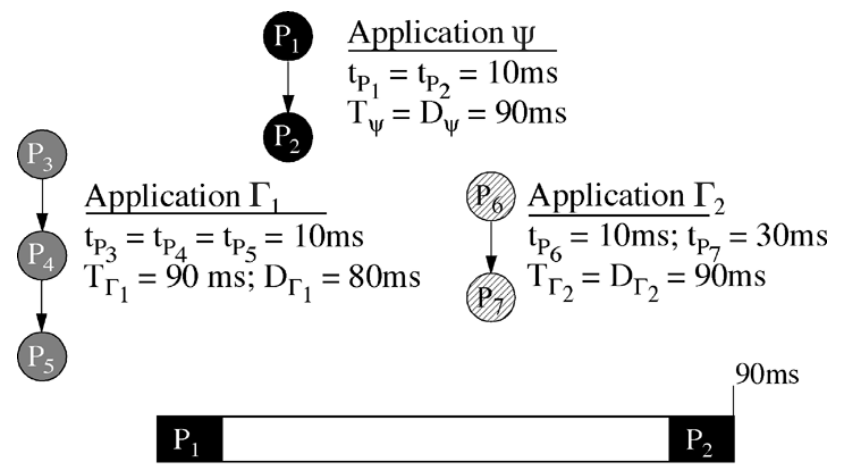

a) Initial system, running application $\Psi$

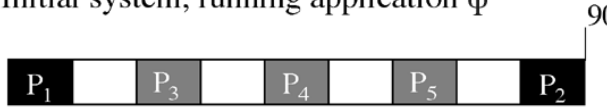

$\mathrm{b}_{1}$ ) Application $\Gamma_{1}$ on top of $\psi: 1^{\text {st }}$ alternative

\begin{tabular}{|l|l|l|l|l|l|l|}
$\mathrm{P}_{1}$ & $\mathrm{P}_{3}$ & & $\mathrm{P}_{4}$ & & $\mathrm{P}_{5}$ & \\
\hline
\end{tabular}

$\mathrm{b}_{2}$ ) Application $\Gamma_{2}$ on top of the $1^{\text {st }}$ alternative: $\mathrm{P}_{7}$ cannot be mapped.

$80 \mathrm{~ms}$

\begin{tabular}{|l|l|l|l|l|l|l}
$\mathrm{P}_{1}$ & $\mathrm{P}_{3}$ & & $\mathrm{P}_{4}$ & & $\mathrm{P}_{5}$ & $\mathrm{P}_{2}$ \\
\hline
\end{tabular}

$\mathrm{c}_{1}$ ) Application $\Gamma_{1}$ on top of $\psi: 2^{\text {nd }}$ alternative

\begin{tabular}{|c|c|c|c|c|}
\hline $\mathrm{P}_{1} \quad \mathrm{P}_{3}$ & 8 & $\mathrm{P}_{4}$ & D. & \\
\hline
\end{tabular}

$c_{2}$ ) Application $\Gamma_{2}$ on top of the $2^{\text {nd }}$ alternative: successful implementation.

\begin{tabular}{|l|l|l|l|ll}
$\mathrm{P}_{1}$ & $\mathrm{P}_{3}$ & & $\mathrm{P}_{4}$ & $\mathrm{P}_{5}$ & $\mathrm{P}_{2}$ \\
\hline
\end{tabular}

$\mathrm{d}_{1}$ ) Application $\Gamma_{1}$ on top of $\psi: 3 \mathrm{r}^{\mathrm{d}}$ alternative

\begin{tabular}{|c|c|c|c|c|}
\hline $\mathrm{P}_{1} \quad \mathrm{P}_{3}$ & P. & P & $\mathrm{P}_{4}$ & $P_{5}$ \\
\hline
\end{tabular}

$\mathrm{d}_{2}$ ) Application $\Gamma_{2}$ on top of the $3^{\text {rd }}$ alternative: successful implementation.

Fig. 5. Application $\Gamma_{2}$ implemented on top of $\Psi$ and $\Gamma_{1}$.

are satisfied, but also there is a good chance that later, new functionality can easily be mapped on the resulted system.

To illustrate the role of mapping and scheduling in the context of an incremental design process, let us consider the example in Fig. 5. For simplicity, we consider an architecture consisting of a single processor. The system is currently running application $\Psi$ [Fig. 5(a)]. At a particular moment application $\Gamma_{1}$ has to be implemented on top of $\Psi$. Three possible implementation alternatives for $\Gamma_{1}$ are depicted in Fig. 5(b $\left.b_{1}\right), 5\left(c_{1}\right)$, and $5\left(d_{1}\right)$. All three are meeting the imposed time constraint for $\Gamma_{1}$. At a later moment, application $\Gamma_{2}$ has to be implemented on the system running $\Psi$ and $\Gamma_{1}$. If $\Gamma_{1}$ has been implemented as shown in Fig. $5\left(b_{1}\right)$, there is no possibility to map application $\Gamma_{2}$ on the given system (in particular, there is no time slack available for process $\mathrm{P}_{7}$ ). If $\Gamma_{1}$ has been implemented as in Fig. 5( $\left.c_{1}\right)$ or $5\left(d_{1}\right), \Gamma_{2}$ can be correctly mapped and scheduled on top of $\Psi$ and $\Gamma_{1}$. There are two aspects which should be highlighted based on this example:

1) If application $\Gamma_{1}$ is implemented like in Fig. $5\left(c_{1}\right)$ or $5\left(\mathrm{~d}_{1}\right)$, it is possible to implement $\Gamma_{2}$ on top of the existing system, without performing any modifications on the implementation of previous applications. This could be the case if, during implementation of $\Gamma_{1}$, the designers have taken into consideration the fact that, in future, an application having the characteristics of $\Gamma_{2}$ will possibly be added to the system. 2) If $\Gamma_{1}$ has been implemented like in Fig. 5( $\left.b_{1}\right), \Gamma_{2}$ can be added to the system only after performing certain modifications on the implementation of $\Gamma_{1}$ and/or $\Psi$. In this case, of course, it is important to perform as few as possible modifications on previous applications, in order to reduce the development costs.

\section{PROBlem Formulation}

As shown in Section II, we capture the functionality of a system as a set of applications. An application $\Gamma$ consists of a set of process graphs $G_{i} \in \Gamma$. For each process $P_{i}$ in a process graph we know the set $\mathcal{N}_{P_{i}}$ of potential nodes on which it could be mapped and its worst-case execution time on each of these nodes. We also know the maximum number of bits to be transmitted by each message. The underlying architecture is as presented in Section II-A. We consider a nonpreemptive static cyclic scheduling policy for both processes and message passing.

Our goal is to map and schedule an application $\Gamma_{\text {current }}$ on a system that already implements a set $\Psi$ of applications, considering the following requirements:

- Requirement a: All constraints on $\Gamma_{\text {current }}$ are satisfied and minimal modifications are performed to the implementation of applications in $\Psi$.

- Requirement $b$ : New applications $\Gamma_{\text {future }}$ can be mapped on top of the resulting system.

We illustrate such an incremental design process in Fig. 6. The product is implemented as a three processor system and its version $N-1$ consists of the set $\Psi$ of two applications (the processes belonging to these applications are represented as white and black disks, respectively). At the current moment, application $\Gamma_{\text {current }}$ is to be added to the system, resulting in version $N$ of the product. However, a new version, $N+1$, is very likely to follow and this fact is to be considered during implementation of $\Gamma_{\text {current }}{ }^{1}$

If it is not possible to map and schedule $\Gamma_{\text {current }}$ without modifying the implementation of the already running applications, we have to change the scheduling and mapping of some applications in $\Psi$. However, even with remapping and rescheduling all applications in $\Psi$, it is still possible that certain constraints are not satisfied. In this case, the hardware architecture has to be changed by, for example, adding a new processor, and the mapping and scheduling procedure for $\Gamma_{\text {current }}$ has to be restarted. In this paper, we will not further elaborate on the aspect of adding new resources to the architecture, but will concentrate on the mapping and scheduling aspects. Thus, we consider that a possible mapping and scheduling of $\Gamma_{\text {current }}$ which satisfies the imposed constraints can be found (with minimizing the modification of the already running applications), and this solution has

\footnotetext{
${ }^{1}$ The design process outlined here also applies when $\Gamma_{\text {current }}$ is a new version of an application $\Gamma_{\text {old }} \in \Psi$. In this case, all the processes and communications belonging to $\Gamma_{\text {old }}$ are eliminated from the running system $\Psi$, before starting the mapping and scheduling of $\Gamma_{\text {current }}$.
} 


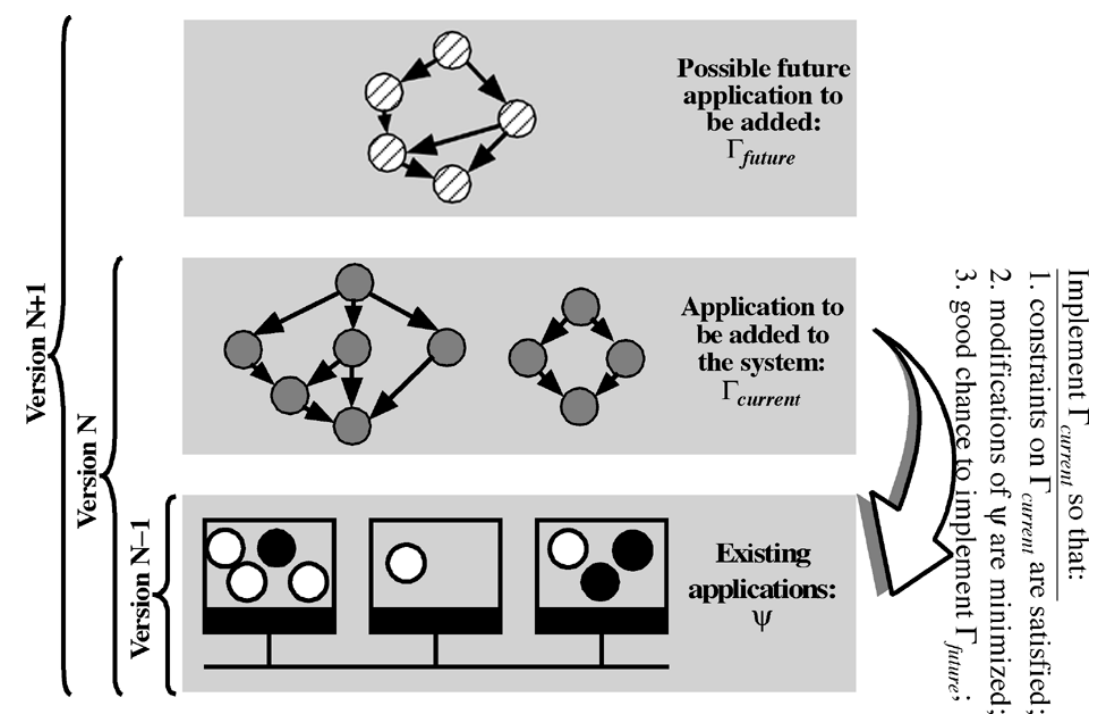

Fig. 6. Incremental design process.

to be further improved in order to facilitate the implementation of future applications.

In order to achieve our goal we need certain information to be available concerning the set of applications $\Psi$ as well as the possible future applications $\Gamma_{\text {future }}$. What exactly we have to know about these applications will be discussed in Section IV. In Section V, we then introduce the quality metrics which will allow us to give a more rigorous formulation of the problem we are going to solve.

The processes in application $\Gamma_{\text {current }}$ can interact with the previously mapped applications $\Psi$ by reading messages generated on the bus by processes in $\Psi$. In this case, the reading process has to be synchronized with the arrival of the message on the bus, which is easy to model as an additional time constraint on the particular receiving process. This constraint is then considered (as any other deadline) during scheduling of $\Gamma_{\text {current }}$.

\section{Characterizing EXISTING AND Future APPliCATIONS}

\section{A. Characterizing the Already Running Applications}

To perform the mapping and scheduling of $\Gamma_{\text {current }}$, the minimum information needed, concerning the already running applications $\Psi$, consists of the local schedule tables for each processor node. Thus, we know the activation time for each process previously mapped on the respective node and its worst-case execution time. As for messages, their length as well as their place in the particular TDMA frame are known.

If the initial attempt to schedule and map $\Gamma_{\text {current }}$ does not succeed, we have to modify the schedule and, possibly, the mapping of applications belonging to $\Psi$, in the hope to find a valid solution for $\Gamma_{\text {current }}$. The goal is to find that minimal modification to the existing system that leads to a correct implementation of $\Gamma_{\text {current }}$. In our context, such a minimal modification means remapping and/or rescheduling a subset $\Omega$ of the old applications, $\Omega \subseteq \Psi$, so that the total cost of reimplementing $\Omega$ is minimized.
Remapping and/or rescheduling a certain application $\Gamma_{i} \in \Psi$ can trigger the need to also perform modifications of one or several other applications because of the dependencies between processes belonging to these applications. In order to capture such dependencies between the applications in $\Psi$, as well as their modification costs, we have introduced a representation called the application graph. We represent a set of applications as a directed acyclic graph $\mathcal{G}(\mathcal{V}, \mathcal{E})$, where each node $\Gamma_{i} \in \mathcal{V}$ represents an application. An edge $e_{i j} \in \mathcal{E}$ from $\Gamma_{i}$ to $\Gamma_{j}$ indicates that any modification to $\Gamma_{i}$ would trigger the need to also remap and/or reschedule $\Gamma_{j}$, because of certain interactions between the applications. ${ }^{2}$ Each application in the graph has an associated attribute specifying if that particular application is allowed to be modified or not (in which case, it is called "frozen"). To those nodes $\Gamma_{i} \in \mathcal{V}$ representing modifiable applications, the designer has associated a cost $R_{\Gamma_{i}}$ of reimplementing $\Gamma_{i}$. Given a subset of applications $\Omega \subseteq \Psi$, the total cost of modifying the applications in $\Omega$ is

$$
R(\Omega)=\sum_{\Gamma_{i} \in \Omega} R_{\Gamma_{i}}
$$

Modifications of an already running application can only be performed if the process graphs corresponding to that application, as well as the related deadlines (which have to be satisfied also after remapping and rescheduling) are available. However, this is not always the case and in such situations that particular application has to be considered frozen.

In Fig. 7, we present the graph corresponding to a set of ten applications. Applications $\Gamma_{6}, \Gamma_{8}, \Gamma_{9}$, and $\Gamma_{10}$, depicted in black, are frozen: no modifications are possible to them. The rest of the applications have the modification cost $R_{\Gamma_{i}}$ depicted on their left. $\Gamma_{7}$ can be remapped/rescheduled with a cost of 20 . If $\Gamma_{4}$ is to be reimplemented, this also requires the modification of $\Gamma_{7}$, with a total cost of 90 . In the case of $\Gamma_{5}$, although

\footnotetext{
2If a set of applications have a circular dependence, such that the modification of any one implies the remapping of all the others in that set, the set will be represented as a single node in the graph.
} 


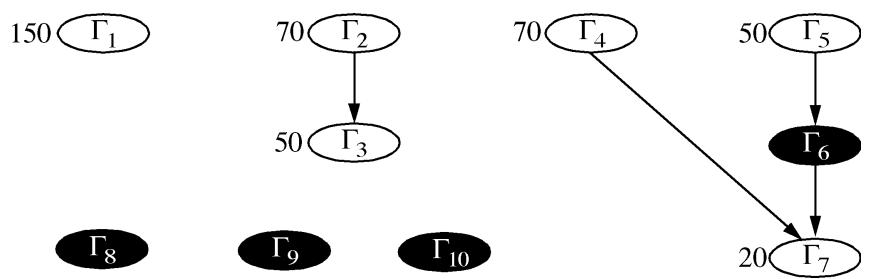

Fig. 7. Characterizing the set of already running applications.

not frozen, no remapping/rescheduling is possible as it would trigger the need to modify $\Gamma_{6}$, which is frozen.

To each application $\Gamma_{i} \in \mathcal{V}$ the designer has associated a $\operatorname{cost} R_{\Gamma_{i}}$ of reimplementing $\Gamma_{i}$. Such a cost can typically be expressed in man-hours needed to perform retesting of $\Gamma_{i}$ and other tasks connected to the remapping and rescheduling of the application. If an application is remapped or rescheduled, it has to be validated again. Such a validation phase is very time consuming. For example, in the automotive industry, the time-to-market in the case of the powertrain unit is 24 months; out of these, 5 months representing more than $20 \%$ are dedicated to validation. In the case of the telematic unit, the time to market is less than one year, while the validation time is two months [38]. However, if an application is not modified during implementation of new functionality, only a small part of the validation tasks have to be reperformed (e.g., integration testing), thus reducing significantly the time-to-market at no additional hardware or development cost.

How to concretely perform the estimation of the modification cost related to an application is beyond the topic of this paper. Several approaches to cost estimation for different phases of the software life cycle have been elaborated and are available in the literature [6], [35]. One of the most influential software cost models is the Constructive Cost Model (COCOMO) [3]. COCOMO is at the core of tools such as REVIC [43] and its newer version SoftEST [44], which can produce cost estimations not only for the total development but also for testing, integration, or modification related retesting of embedded software. The results of such estimations can be used by the designer as the cost metrics assigned to the nodes of an application graph.

In general, it can be the case that several alternative costs are associated to a certain application, depending on the particular modification performed. Thus, for example, we can have a certain cost if processes are only rescheduled, and another one if they are also remapped on an alternative node. For different modification alternatives considered during design space exploration, the corresponding modification cost has to be selected. In order to keep the discussion reasonably simple, we present the case with one single modification cost associated with an application. However, the generalization for several alternative modification costs is straightforward.

\section{B. Characterizing Future Applications}

What do we suppose to know about the family $\Gamma_{\text {future }}$ of applications which do not exist yet? Given a certain limited application area (e.g., automotive electronics), it is not unreasonable to assume that, based on the designers' previous experience, the nature of expected future functions to be implemented, profiling of previous applications, available incomplete designs for future versions of the product, etc., it is possible to characterize the family of applications which possibly could be added to the current implementation. This is an assumption which is basic for the concept of incremental design. Thus, we consider that, with respect to the future applications, we know the set $S_{t}=\left\{t_{\min }, \ldots t_{i}, \ldots t_{\max }\right\}$ of possible worst-case execution times for processes, and the set $S_{b}=\left\{b_{\min }, \ldots b_{i}, \ldots b_{\max }\right\}$ of possible message sizes. We also assume that over these sets we know the distributions of probability $f_{S_{t}}(t)$ for $t \in S_{t}$ and $f_{S_{b}}(b)$ for $b \in S_{b}$. For example, we might have predicted possible worst-case execution times of different processes in future applications $S_{t}=\{50,100,200,300,500 \mathrm{~ms}\}$. If there is a higher probability of having processes of $100 \mathrm{~ms}$, and a very low probability of having processes of $300 \mathrm{~ms}$ and $500 \mathrm{~ms}$, then our distribution function $f_{S_{t}}(t)$ could look like this: $f_{S_{t}}(50)=$ $0.20, f_{S_{t}}(100)=0.50, f_{S_{t}}(200)=0.20, f_{S_{t}}(300)=0.05$, and $f_{S_{t}}(500)=0.05$.

Another information is related to the period of process graphs which could be part of future applications. In particular, the smallest expected period $T_{\min }$ is assumed to be given, together with the expected necessary processor time $t_{\text {need }}$, and bus bandwidth $b_{\text {need }}$, inside such a period $T_{\min }$. As will be shown later, this information is treated in a flexible way during the design process and is used in order to provide a fair distribution of available resources.

The execution times in $S_{t}$, as well as $t_{\text {need }}$, are considered relative to the slowest node in the system. All the other nodes are characterized by a speedup factor relative to this slowest node. A normalization with these factors is performed when computing the metrics $\mathrm{C}_{1}^{P}$ and $\mathrm{C}_{2}^{P}$ introduced in Section $\mathrm{V}$.

\section{Quality Metrics AND OBJective Function}

A designer will be able to map and schedule an application $\Gamma_{\text {future }}$ on top of a system implementing $\Psi$ and $\Gamma_{\text {current }}$ only if there are sufficient resources available. In our case, the resources are processor time and the bandwidth on the bus. In the context of a nonpreemptive static scheduling policy, having free resources translates into having free time slots on the processors and having space left for messages in the bus slots. We call these free slots of available time on the processor or on the bus, slack. It is to be noted that the total quantity of computation and communication power available on our system after we have mapped and scheduled $\Gamma_{\text {current }}$ on top of $\Psi$ is the same regardless of the mapping and scheduling policies used. What depends on the mapping and scheduling strategy is the distribution of slacks along the time line and the size of the individual slacks. It is exactly this size and distribution of the slacks that characterizes the quality of a certain design alternative from the point of view of flexibility for future upgrades. In this section, we introduce two criteria in order to reflect the degree to which one design alternative meets the requirement $b$ presented in Section III. For each criterion we provide metrics which quantify the degree to which the criterion is met. The first criterion reflects how well the resulted slack sizes fit a future application, and the second criterion expresses how well the slack is distributed in time. 


\section{A. Slack Sizes (The First Criterion)}

The slack sizes resulted after implementation of $\Gamma_{\text {current }}$ on top of $\Psi$ should be such that they best accommodate a given family of applications $\Gamma_{\text {future, }}$ characterized by the sets $S_{t}, S_{b}$ and the probability distributions $f_{S_{t}}$ and $f_{S_{b}}$, as outlined in Section IV-B.

Let us go back to the example in Fig. 5 where $\Gamma_{1}$ is what we now call $\Gamma_{\text {current }}$, while $\Gamma_{2}$, to be later implemented on top of $\Psi$ and $\Gamma_{1}$, is $\Gamma_{\text {future }}$. This $\Gamma_{\text {future }}$ consists of the two processes $P_{6}$ and $P_{7}$. It can be observed that the best configuration from the point of view of accommodating $\Gamma_{\text {future }}$, taking in consideration only slack sizes, is to have a contiguous slack after implementation of $\Gamma_{\text {current }}$ [Fig. $\left.5\left(\mathrm{~d}_{1}\right)\right]$. However, in reality, it is almost impossible to map and schedule the current application such that a contiguous slack is obtained. Not only is it impossible, but it is also undesirable from the point of view of the second design criterion, to be discussed next. However, as we can see from Fig. $5\left(b_{1}\right)$, if we schedule $\Gamma_{\text {current }}$ such that it fragments too much the slack, it is impossible to fit $\Gamma_{\text {future }}$ because there is no slack that can accommodate process $P_{7}$. A situation as the one depicted in Fig. 5(c $\left.c_{1}\right)$ is desirable, where the resulted slack sizes are adapted to the characteristics of the $\Gamma_{\text {future }}$ application.

In order to measure the degree to which the slack sizes in a given design alternative fit the future applications, we provide two metrics: $\mathrm{C}_{1}^{P}$ and $\mathrm{C}_{1}^{m}$. $\mathrm{C}_{1}^{P}$ captures how much of the largest future application that theoretically could be mapped on the system can be mapped on top of the current design alternative. $\mathrm{C}_{1}^{m}$ is similar relative to the slacks in the bus slots.

How does the largest future application which theoretically could be mapped on the system look like? The total processor time and bus bandwidth available for this largest future application is the total slack available on the processors and bus, respectively, after implementing $\Gamma_{\text {current }}$. Process and message sizes of this hypothetical largest application are determined knowing the total size of the available slack, and the characteristics of the future applications as expressed by the sets $S_{t}$ and $S_{b}$, and the probability distributions $f_{S_{t}}$ and $f_{S_{b}}$. Let us consider, for example, that the total slack size on the processors is of $2800 \mathrm{~ms}$ and the set of possible worst-case execution times is $S_{t}=\{50,100,200,300,500 \mathrm{~ms}\}$. The probability distribution function $f_{S_{t}}$ is defined as follows: $f_{S_{t}}(50)=0.20$, $f_{S_{t}}(100)=0.50, f_{S_{t}}(200)=0.20, f_{S_{t}}(300)=0.05$, and $f_{S_{t}}(500)=0.05$. Under these circumstances, the largest hypothetical future application will consist of 20 processes: 10 processes (half of the total, $f_{t}(100)=0.50$ ) with a worst-case execution time of $100 \mathrm{~ms}, 4$ processes with $50 \mathrm{~ms}, 4$ with $200 \mathrm{~ms}$, one with 300 and one with $500 \mathrm{~ms}$.

After we have determined the number of processes of this largest hypothetical $\Gamma_{\text {future }}$ and their worst-case execution times, we apply a bin-packing algorithm [22] using the best-fit policy in which we consider processes as the objects to be packed, and the available slacks as containers. The total execution time of processes which are left unpacked, relative to the total execution time of the whole process set, gives the $\mathrm{C}_{1}^{P}$ metric. The same is the case with the metric $\mathrm{C}_{1}^{m}$, but applied to message sizes and available slacks in the bus slots.
Let us consider the example in Fig. 5 and suppose a hypothetical $\Gamma_{\text {future }}$ consisting of two processes like those of application $\Gamma_{2}$. For the design alternatives in Fig. $5\left(c_{1}\right)$ and $5\left(d_{1}\right), C_{1}^{P}=0 \%$ (both alternatives are perfect from the point of view of slack sizes). For the alternative in Fig. 5( $\left.\mathrm{b}_{1}\right)$, however, $\mathrm{C}_{1}^{P}=30 / 40=75 \%$-the worst-case execution time of $P_{7}$ (which is left unpacked) relative the total execution time of the two processes.

\section{B. Distribution of Slacks (The Second Criterion)}

In Section V-A, we defined a metric which captures how well the sizes of the slacks fit a possible future application. A similar metric is needed to characterize the distribution of slacks over time.

Let $P_{i}$ be a process with period $T_{P_{i}}$ that belongs to a future application, and $M\left(P_{i}\right)$ the node on which $P_{i}$ will be mapped. The worst-case execution time of $P_{i}$ is $t_{P_{i}}^{M\left(P_{i}\right)}$. In order to schedule $P_{i}$ we need a slack of size $t_{P_{i}}^{M\left(P_{i}\right)}$ that is available periodically, within a period $T_{P_{i}}$, on processor $M\left(P_{i}\right)$. If we consider a group of processes with period $T$, which are part of $\Gamma_{\text {future }}$, in order to implement them, a certain amount of slack is needed that is available periodically, with a period $T$, on the nodes implementing the respective processes.

During implementation of $\Gamma_{\text {current }}$ we aim for a slack distribution such that the future application with the smallest expected period $T_{\min }$ and with the necessary processor time $t_{\text {need }}$, and bus bandwidth $b_{\text {need }}$, can be accommodated (see Section IV-B).

Thus, for each node, we compute the minimum periodic slack, inside a $T_{\min }$ period. By summing these minima, we obtain the slack which is available periodically to $\Gamma_{\text {future }}$ This is the $\mathrm{C}_{2}^{P}$ metric. The $\mathrm{C}_{2}^{m}$ metric characterizes the minimum periodically available bandwidth on the bus and it is computed in a similar way.

In Fig. 8 we consider an example with $T_{\min }=120 \mathrm{~ms}, t_{\text {need }}=$ $90 \mathrm{~ms}$, and $b_{\text {need }}=65 \mathrm{~ms}$. The length of the schedule table of the system implementing $\Psi$ and $\Gamma_{\text {current }}$ is $360 \mathrm{~ms}$ (in Section VI we will elaborate on the length of the global schedule table). Thus, we have to investigate three periods of length $T_{\min }$ each. The system consists of three nodes. Let us consider the situation in Fig. 8(a). In the first period, Period 0 , there are $40 \mathrm{~ms}$ of slack available on Node $_{1}$, in the second period $80 \mathrm{~ms}$, and in the third period no slack is available on Node $_{1}$. Thus, the total slack a future application of period $T_{\min }$ can use on $\operatorname{Node}_{1}$ is $\min (40,80,0)=0 \mathrm{~ms}$. Neither can $\mathrm{Node}_{2}$ provide slack for this application, as in Period 1 there is no slack available. However, on $\mathrm{Node}_{3}$ there are at least $40 \mathrm{~ms}$ of slack available in each period. Thus, with the configuration in Fig. 8(a) we have $\mathrm{C}_{2}^{P}=40 \mathrm{~ms}$, which is not sufficient to accommodate $t_{\text {need }}=90 \mathrm{~ms}$. The available periodic slack on the bus is also insufficient: $\mathrm{C}_{2}^{m}=60 \mathrm{~ms}<b_{\text {need }}$. However, in the situation presented in Fig. 8(b), we have $\mathrm{C}_{2}^{P}=120 \mathrm{~ms}>t_{\text {need }}$, and $\mathrm{C}_{2}^{m}=90 \mathrm{~ms}>b_{\text {need }}$.

\section{Objective Function and Exact Problem Formulation}

In order to capture how well a certain design alternative meets the requirement (b) stated in Section III, the metrics discussed before are combined in an objective function, as follows:

$$
\begin{aligned}
C=w_{1}^{P}\left(\mathrm{C}_{1}^{P}\right)^{2}+w_{1}^{m}\left(\mathrm{C}_{1}^{m}\right)^{2}+w_{2}^{P} \max \left(0, t_{\text {need }}-\mathrm{C}_{2}^{P}\right) \\
+w_{2}^{m} \max \left(0, b_{\text {need }}-\mathrm{C}_{2}^{m}\right)
\end{aligned}
$$




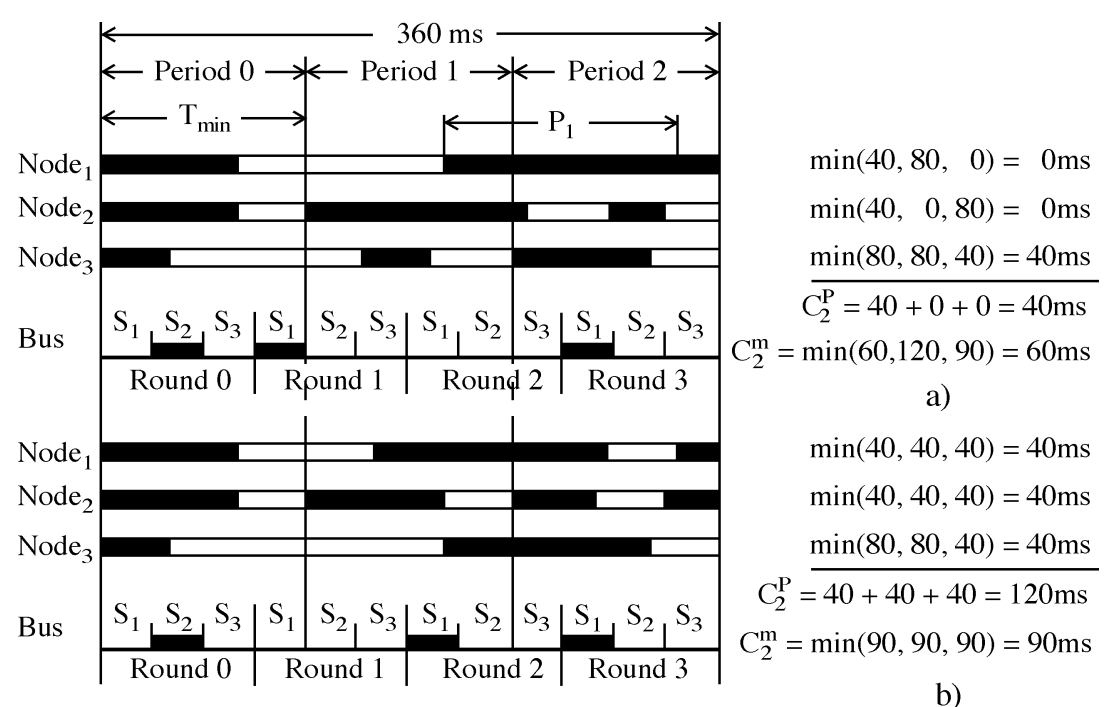

Time slots occupied by $\psi$ and $\Gamma_{\text {current }}$

Slack

b)

Fig. 8. Example for the second design criterion.

where the metric values introduced in the previous section are weighted by the constants $w_{1}^{P}, w_{2}^{P}, w_{1}^{m}$, and $w_{2}^{m}$. Our mapping and scheduling strategy will try to minimize this function.

The first two terms measure how well the resulted slack sizes fit to a future application (the first criterion), while the second two terms reflect the distribution of slacks (the second criterion). In order to obtain a balanced solution, that favors a good fitting both on the processors and on the bus, we have used the squares of the metrics.

We call a valid solution, that mapping and scheduling which satisfies all the design constraints (in our case the deadlines) and meets the second criterion $\left(\mathrm{C}_{2}^{P} \geq t_{\text {need }}\right.$ and $\left.\mathrm{C}_{2}^{m} \geq b_{\text {need }}\right){ }^{3}$

At this point we can give an exact formulation of our problem. Given an existing set of applications $\Psi$ which are already mapped and scheduled, and an application $\Gamma_{\text {current }}$ to be implemented on top of $\Psi$, we are interested to find the subset $\Omega \subseteq \Psi$ of old applications to be remapped and rescheduled such that we produce a valid solution for $\Gamma_{\text {current }} \cup \Omega$ and the total cost of modification $R(\Omega)$ is minimized. Once such a set $\Omega$ of applications is found, we are interested to optimize the implementation of $\Gamma_{\text {current }} \cup \Omega$ such that the objective function $C$ is minimized, considering a family of future applications characterized by the sets $S_{t}$ and $S_{b}$, the functions $f_{S_{t}}$ and $f_{S_{b}}$ as well as the parameters $T_{\min }, t_{\text {need }}$, and $b_{\text {need }}$.

A mapping and scheduling strategy based on this problem formulation is presented in the following section.

\section{MAPPING AND SCHEdULING STRATEGY}

As shown in the algorithm in Fig. 9, our mapping and scheduling strategy (MS) consists of two steps. In the first step, we try to obtain a valid solution for the mapping and scheduling of $\Gamma_{\text {current }} \cup \Omega$ so that the modification cost $R(\Omega)$ is minimized. Starting from such a solution, the second step iteratively improves the design in order to minimize the objective function

\footnotetext{
${ }^{3}$ This definition of a valid solution can be relaxed by imposing only the satisfaction of deadlines. In this case, the algorithm in Fig. 9 will look after a solution which satisfies the deadlines and $R(\Omega)$ is minimized; the additional second criterion is, in this case, only considered optionally.
}

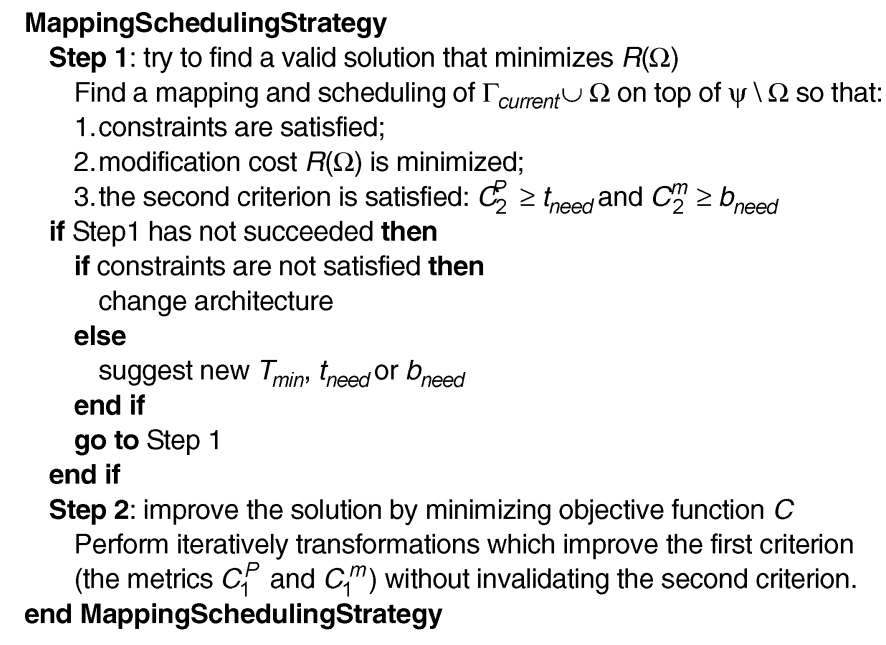

Fig. 9. Mapping and scheduling strategy (MS).

$C$. In the context in which the second criterion is satisfied after the first step, improving the cost function during the second step aims at minimizing the value of $w_{1}^{P}\left(\mathrm{C}_{1}^{P}\right)^{2}+w_{1}^{m}\left(\mathrm{C}_{1}^{m}\right)^{2}$.

If the first step has not succeeded in finding a solution such that the imposed time constraints are satisfied, this means that there are not sufficient resources available to implement the application $\Gamma_{\text {current }}$. Thus, modifications of the system architecture have to be performed before restarting the mapping and scheduling procedure. If, however, the timing constraints are met but the second design criterion is not satisfied, a larger $T_{\min }$ (smallest expected period of a future application, see Section IV-B) or smaller values for $t_{\text {need }}$ and/or $b_{\text {need }}$ are suggested to the designer. This, of course, reduces the frequency of possible future applications and the amount of processor and bus resources available to them.

In Section VI-A, we briefly discuss the basic mapping and scheduling algorithm we have used in order to generate an initial solution. The heuristic used to iteratively improve the design with regard to the first and the second design criteria is presented in Section VI-B. In Section VI-C, we describe three alternative 


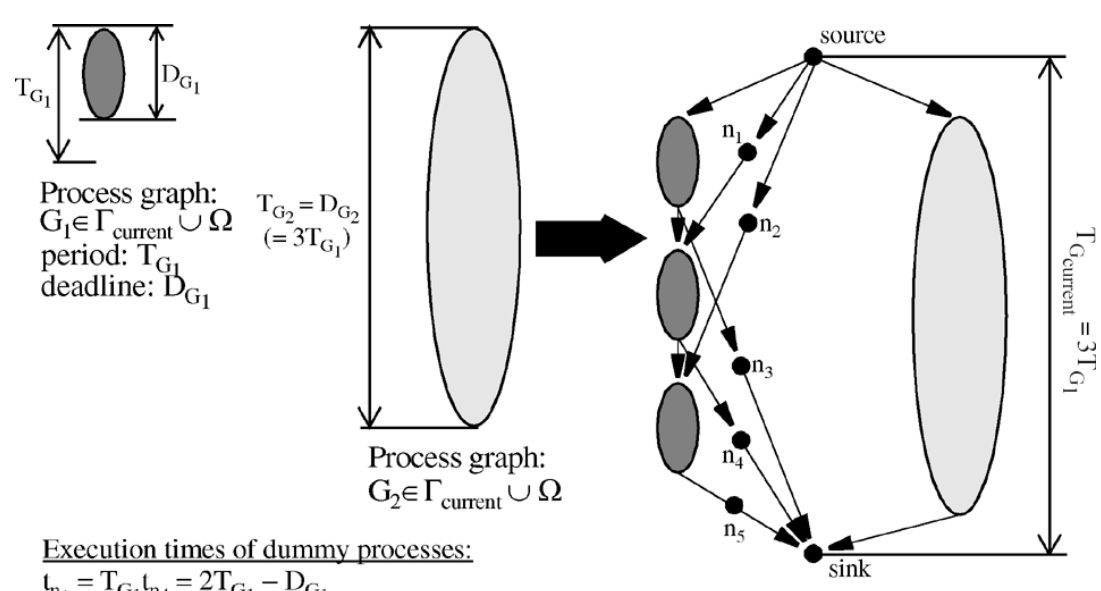

$$
\begin{aligned}
& \mathrm{t}_{\mathrm{n}_{1}}=\mathrm{T}_{\mathrm{G}_{1}} \mathrm{t}_{\mathrm{n}_{4}}=2 \mathrm{~T}_{\mathrm{G}_{1}}-\mathrm{D}_{\mathrm{G}_{1}} \\
& \mathrm{t}_{\mathrm{n}_{2}}=2 \mathrm{~T}_{\mathrm{G}_{1}} \mathrm{t}_{\mathrm{n}_{5}}=\mathrm{T}_{\mathrm{G}_{1}}-\mathrm{D}_{\mathrm{G}_{1}} \\
& \mathrm{t}_{\mathrm{n}_{3}}=3 \mathrm{~T}_{\mathrm{G}_{1}}-\mathrm{D}_{\mathrm{G}_{1}} \mathrm{t}_{\text {source }}=\mathrm{t}_{\text {sink }}=0
\end{aligned}
$$

Merged process graph $\mathrm{G}_{\text {current }}$ : period $=$ deadline $=3 \mathrm{~T}_{\mathrm{G}_{1}}$

Fig. 10. Graph merging.

heuristics that can be used during the first step in order to find the optimal subset of applications to be modified.

\section{A. The Initial Mapping and Scheduling}

As shown in Fig. 11, the first step of MS consists of an iteration that tries different subsets $\Omega \subseteq \Psi$ with the intention to find that subset $\Omega=\Omega_{\min }$ of old applications to be remapped and rescheduled which produces a valid solution for $\Gamma_{\text {current }} U$ $\Omega$ such that $R(\Omega)$ is minimized. Given a subset $\Omega$, the InitialMappingScheduling function (IMS) constructs a mapping and a schedule for the applications $\Gamma_{\text {current }} \cup \Omega$ on top of $\Psi \backslash \Omega$, that meets the deadlines, without worrying about the two criteria introduced in Section V.

The IMS is a classical mapping and scheduling algorithm for which we have used as a starting point the heterogeneous critical path (HCP) algorithm, introduced in [13]. HCP is based on a list scheduling approach [4]. We have modified the HCP algorithm in three main regards.

1) We consider that mapping and scheduling does not start with an empty system but a system on which a certain number of processes already are mapped.

2) Messages are scheduled into bus slots according to the TDMA protocol. The TDMA-based message scheduling technique has been presented by us in [8].

3) As a priority function for list scheduling we use, instead of the critical path $(\mathrm{CP})$ priority function employed in [13], the modified partial critical path (MPCP) function introduced by us in [8]. MPCP takes into consideration the particularities of the communication protocol for calculation of communication delays. These delays are not estimated based only on the message length, but also on the time when slots assigned to the particular node which generates the message will be available.

For the example in Fig. 4, our initial mapping and scheduling algorithm will be able to produce the optimal solution with a schedule length of $48 \mathrm{~ms}$.

However, before performing the effective mapping and scheduling with IMS, two aspects have to be addressed. First, the process graphs $G_{i} \in \Gamma_{\text {current }} \cup \Omega$ have to be merged into a single graph $G_{\text {current }}$, by unrolling of process graphs and
Step 1: try to find a valid solution that minimizes $R(\Omega)$

$$
\begin{aligned}
& \Omega=\varnothing \\
& \text { repeat } \\
& \text { succeeded }=\text { InitialMappingScheduling }\left(\psi \backslash \Omega, \Gamma_{\text {current }} \cup \Omega\right) \\
& \text {-- compute ASAP-ALAP intervals for all processes } \\
& \operatorname{ASAP}\left(\Gamma_{\text {current }} \cup \Omega\right) \text {; } \operatorname{ALAP}\left(\Gamma_{\text {current }} \cup \Omega\right) \\
& \text { if succeeded then-- if time constraints are satisfied } \\
& \text {-- design transformations in order to satisfy } \\
& \text {-- the second design criterion } \\
& \text { repeat } \\
& \text {-- find set of moves with the highest potential to } \\
& \text {-- maximize } C_{2}^{P} \text { or } C_{2}^{m} \\
& \text { move_set }=\text { PotentialMoveC }{ }_{2}^{\mathrm{P}}\left(\Gamma_{\text {current }} \cup \Omega\right) \cup \\
& \text { PotentialMoveC } \mathrm{C}_{2}^{\mathrm{m}}\left(\Gamma_{\text {current }} \cup \Omega\right) \\
& \text {-- select and perform move which improves most } C_{2} \\
& \text { move }=\text { SelectMoveC }{ }_{2} \text { (move_set); Perform (move) } \\
& \text { succeeded }=C_{2}^{P} \geq t_{\text {need }} \text { and } C_{2}^{m} \geq b_{\text {need }} \\
& \text { until succeeded or maximum number of iterations reached } \\
& \text { end if } \\
& \text { if succeeded and } R(\Omega) \text { smallest so far then } \\
& \Omega_{\text {valid }}=\Omega ; \text { solution }_{\text {valid }}=\text { solution }_{\text {current }} \\
& \text { end if } \\
& \Omega=\operatorname{NextSubset}(\Omega) \text {-- try another subset } \\
& \text { until termination condition }
\end{aligned}
$$

Step 2: improve the solution by minimizing objective function $C$ solution $_{\text {current }}=$ solution $_{\text {valid; }} ; \Omega_{\min }=\Omega_{\text {valid }}$

-- design transformations in order to satisfy the first design criterion repeat

-- find set of moves with highest potential to minimize $C_{1}^{P}$ or $C_{1}^{m}$ move_set $=$ PotentialMoveC ${ }_{1}^{P}\left(\Gamma_{\text {current }} \cup \Omega_{\min }\right) \cup$

PotentialMoveC ${ }_{2}^{m}\left(\Gamma_{\text {current }} \cup \Omega_{\text {mip }}\right)$

-- select move which improve $w_{1}^{P}\left(C_{1}^{P}\right)^{2}+w_{1}^{m}\left(C_{1}^{m}\right)^{2}$,

-- and does not invalidate the second criterion

move $=\mathrm{SelectMoveC}_{1}($ move_set $) ;$ Perform $($ move $)$

until $w_{1}^{P}\left(C_{1}^{P}\right)^{2}+w_{1}^{m}\left(C_{1}^{m}\right)^{2}$ has not changed or

maximum number of iterations reached

Fig. 11. Steps 1 and 2 of the mapping and scheduling strategy in Fig. 9.

insertion of dummy nodes as shown in Fig. 10. The period $T_{G_{\text {current }}}$ of $G_{\text {current }}$ is equal to the least common multiplier of the periods $T_{G_{i}}$ of the graphs $G_{i}$. Dummy nodes (depicted as black disks in Fig. 10) represent processes with a certain execution time but that are not to be mapped to any processor 
or bus. In addition, we have to consider during scheduling the mismatch between the periods of the already existing system and those of the current application. The schedule table into which we would like to schedule $G_{\text {current }}$ has a length of $T_{\Psi \backslash \Omega}$ which is the global period of the system $\Psi$ after extraction of the applications in $\Omega$. However, the period $T_{\text {current }}$ of $G_{\text {current }}$ can be different from $T_{\Psi \backslash \Omega}$. Thus, before scheduling $G_{\text {current }}$ into the existing schedule table, the schedule table is expanded to the least common multiplier of the two periods. A similar procedure is followed in the case $T_{\text {current }}>T_{\Psi \backslash \Omega}$.

\section{B. Iterative Design Transformations}

Once IMS has produced a mapping and scheduling which satisfies the timing constraints, the next goal of Step 1 is to improve the design in order to satisfy the second-design criterion $\left(\mathrm{C}_{2}^{P} \geq t_{\text {need }}\right.$ and $\left.\mathrm{C}_{2}^{m} \geq b_{\text {need }}\right)$. During the second step, the design is then further transformed with the goal of minimizing the value of $w_{1}^{P}\left(\mathrm{C}_{1}^{P}\right)^{2}+w_{1}^{m}\left(\mathrm{C}_{1}^{m}\right)^{2}$, according to the requirements of the first criterion, without invalidating the second criterion achieved in the first step. In both steps, we iteratively improve the design using a transformational approach. These successive transformations are performed inside the (innermost) repeat loops of the first and second step, respectively (Fig. 11). A new design is obtained from the current one by performing a transformation called move. We consider the following two categories of moves:

1) moving a process to a different slack found on the same node or on a different node;

2) moving a message to a different slack on the bus.

In order to eliminate those moves that will lead to an infeasible design (that violates deadlines), we do as follows. For each process $P_{i}$, we calculate the $\operatorname{ASAP}\left(P_{i}\right)$ and $\operatorname{ALAP}\left(P_{i}\right)$ times considering the resources of the given hardware architecture. $\operatorname{ASAP}\left(P_{i}\right)$ is the earliest time $P_{i}$ can start its execution, while $\operatorname{ALAP}\left(P_{i}\right)$ is the latest time $P_{i}$ can start its execution without causing the application to miss its deadline. When moving $P_{i}$ we will consider slacks on the target processor only inside the $\left[\operatorname{ASAP}\left(P_{i}\right), \operatorname{ALAP}\left(P_{i}\right)\right]$ interval. The same reasoning holds for messages, with the addition that a message can only be moved to slacks belonging to a slot that corresponds to the sender node (see Section II-A). Any violation of the data-dependency constraints caused by a move is rectified by shifting processes or messages concerned in an appropriate way. If such a shift produces a deadline violation, the move is rejected.

At each step, our heuristic tries to find those moves that have the highest potential to improve the design. For each iteration a set of potential moves is selected by the PotentialMoveX functions. SelectMoveX then evaluates these moves with regard to the respective metrics and selects the best one to be performed. We now briefly discuss the four PotentialMoveX functions with the corresponding moves.

1) PotentialMove $C_{2}^{P}$ and PotentialMove $C_{2}^{m}$ : Consider Fig. 8(a). In Period 2 on Node $_{1}$ there is no available slack. However, if we move process $P_{1}$ with $40 \mathrm{~ms}$ to the left into $P e$ riod 1, as depicted in Fig. 8(b), we create a slack in Period 2 and the periodic slack on node $N_{1}$ will be $\min (40,40,40)=40 \mathrm{~ms}$, instead of $0 \mathrm{~ms}$.
Potential moves aimed at improving the metric $\mathrm{C}_{2}^{P}$ will be the shifting of processes inside their [ASAP, ALAP] interval in order to improve the periodic slack. The move can be performed on the same node or to the less loaded nodes. The same is true for moving messages in order to improve the metric $\mathrm{C}_{2}^{m}$. For the improvement of the periodic bandwidth on the bus, we also consider movement of processes, trying to place the sender and receiver of a message on the same processor and, thus, reducing the bus load.

2) PotentialMove $C_{1}^{P}$ and PotentialMove $C_{1}^{m}$ : The moves suggested by these two functions aim at improving the $\mathrm{C}_{1}$ metric through reducing the slack fragmentation. The heuristic is to evaluate only those moves that iteratively eliminate the smallest slack in the schedule. Let us consider the example in Fig. 12 where we have three applications mapped on a single processor: $\Psi$, consisting of $P_{1}$ and $P_{2}, \Gamma_{\text {current }}$, having processes $P_{3}, P_{4}$, and $P_{5}$, and $\Gamma_{\text {future }}$, with $P_{6}, P_{7}$, and $P_{8}$. Fig. 12 presents three possible schedules; processes are depicted with rectangles, the width of a rectangle representing the worst-case execution time of that process. The PotentialMoveC func- $_{1}$ tions start by identifying the smallest slack in the schedule table. In Fig. 12(a), the smallest slack is the slack between $P_{1}$ and $P_{3}$. Once the smallest slack has been identified, potential moves are investigated which either remove or enlarge the slack. For example, the slack between $P_{1}$ and $P_{3}$ can be removed by attaching $P_{3}$ to $P_{1}$, and it can be enlarged by moving $P_{3}$ to the right in the schedule table. Moves that remove the slack are considered only if they do not lead to an invalidation of the second design criterion, measured by the $\mathrm{C}_{2}$ metric improved in the previous step (see Fig. 11, Step 1). Also, the slack can be enlarged only if it does not create, as a result, other unusable slack. A slack is unusable if it cannot hold the smallest object of the future application, in our case $P_{6}$. In Fig. 12(a), the slack can be removed by moving $P_{3}$ such that it starts from time 20, immediately after $P_{1}$, and it can be enlarged by moving $P_{3}$ so that it starts from 30,40 , or 50 (considering an increment which here was set by us to 10 , the size of $P_{6}$, the smallest object in $\Gamma_{\text {future }}$ ). For each move, the improvement on the $\mathrm{C}_{1}$ metric is calculated, and that move is selected by the SelectMoveC $C_{1}$ function to be performed, which leads to the largest improvement on $\mathrm{C}_{1}$ (which means the smallest value). For all the previously considered moves of $P_{3}$, we are not able to map $P_{8}$ which represents $50 \%$ of the $\Gamma_{\text {future }}$, therefore, $\mathrm{C}_{1}=50 \%$. Consequently, we can perform any of the mentioned moves, and our algorithm selects the first one investigated, the move to start $P_{3}$ from 20, thus removing the slack. As a result of this move, the new schedule table is the one in Fig. 12(b). In the next call of the PotentialMove $\mathrm{C}_{1}$ function, the slack between $P_{5}$ and $P_{2}$ is identified as the smallest slack. Out of the potential moves that eliminate this slack, listed in Fig. 12 for case $\mathrm{b}$, several lead to $\mathrm{C}_{1}=0 \%$, the largest improvement. SelectMoveC ${ }_{1}$ selects moving $P_{5}$ to start from 90 , and thus we are able to map process $P_{8}$ of the future application, leading to a successful implementation in Fig. 12(c).

The previous example has only illustrated movements of processes. Similarly, in PotentialMoveC ${ }_{1}^{m}$, we also consider moves of messages in order to improve $\mathrm{C}_{1}^{m}$. However, the movement of messages is restricted by the TDMA bus access 


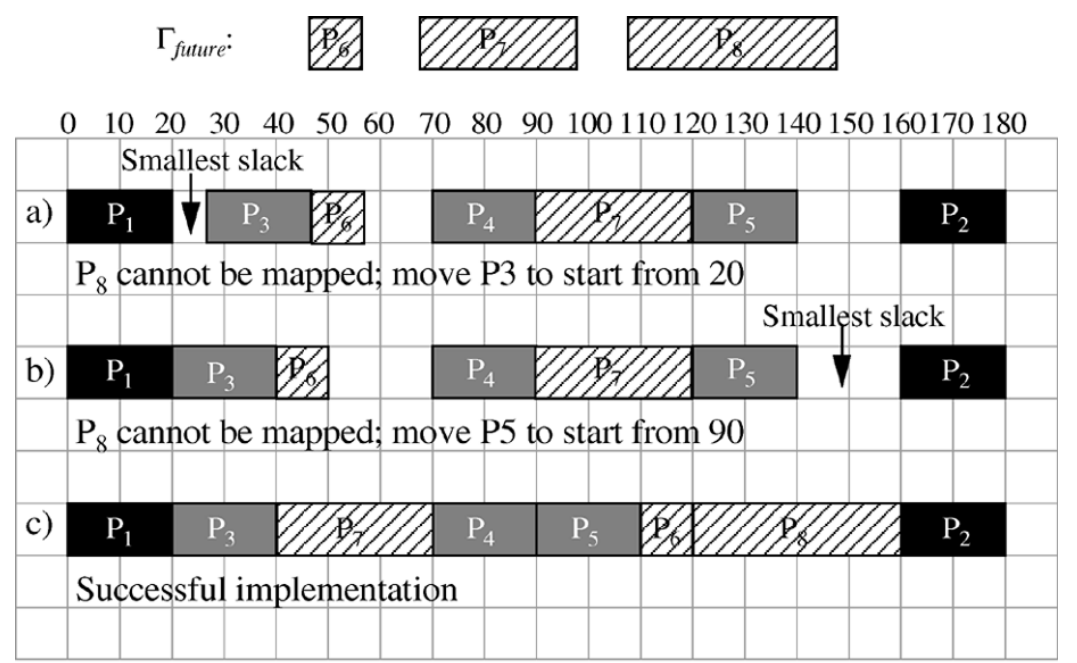

a)

Smallest slack: between $P_{1}$ and $P_{3}$ Smallest slack: between $P_{5}$ and $P_{2}$ Potential moves: $\mathrm{P}_{3}$ starting at 20 , Potential moves: $\mathrm{P}_{4}$ to $40 / 37.5 \%, 50 /$ having $\mathrm{C}_{1}^{\mathrm{P}}=50 \%$ (denoted with $20 / 37.5 \%, 60 / 37.5 \%, 80 / 37.5 \%$, 90/ $50 \%), 30 / 50 \%, 40 / 50 \%, 50 / 50 \%$.

Selected move: $\mathrm{P} 3$ to 20 , with $\mathrm{C}_{1}^{\mathrm{P}}=50 \%$.

Fig. 12. Successive steps with potential moves for improving $C_{1}$.

scheme, such that a message can only be moved into a slot corresponding to the node on which it is generated.

\section{Minimizing the Total Modification Cost}

The first step of our mapping and scheduling strategy, described in Fig. 11, iterates on successive subsets $\Omega$ searching for a valid solution which also minimizes the total modification cost $R(\Omega)$. As a first attempt, the algorithm searches for a valid implementation of $\Gamma_{\text {current }}$ without disturbing the existing applications $(\Omega=\varnothing)$. If no valid solution is found, successive subsets $\Omega$ produced by the function NextSubset are considered, until a termination condition is met. The performance of the algorithm, in terms of runtime and quality of the solutions produced, is strongly influenced by the strategy employed for the function NextSubset and the termination condition. They determine how the design space is explored while testing different subsets $\Omega$ of applications. In the following we present three alternative strategies. The first two can be considered as situated at opposite extremes: The first one is potentially very slow but produces the optimal result while the second is very fast and possibly low quality. The third alternative is a heuristic able to produce good quality results in relatively short time, as will be demonstrated by the experimental results presented in Section VII.

1) Exhaustive Search (ES): In order to find $\Omega_{\min }$, the simplest solution is to try successively all the possible subsets $\Omega \subset$ $\Psi$. These subsets are generated in the ascending order of the total modification cost, starting from $\varnothing$. The termination condition is fulfilled when the first valid solution is found or no new subsets are to be generated. Since the subsets are generated in ascending order, according to their cost, the subset $\Omega$ that first produces a valid solution is also the subset with the minimum modification cost.

The generation of subsets is performed according to the graph $\mathcal{G}$ that characterizes the existing applications (see Section IV-A). Finding the next subset $\Omega$, starting from the current one, is achieved by a branch and bound algorithm that, in the worst case, grows exponentially in time with the number of applications. For the example in Fig. 7, the call to NextSubset $(\varnothing)$ will generate $\Omega=\left\{\Gamma_{7}\right\}$ which has the smallest nonzero modification cost $R\left(\left\{\Gamma_{7}\right\}\right)=20$. The next generated subsets, in order, together with their corresponding total modification cost are: $R\left(\left\{\Gamma_{3}\right\}\right)=50, R\left(\left\{\Gamma_{3}, \Gamma_{7}\right\}\right)=70$, $R\left(\left\{\Gamma_{4}, \Gamma_{7}\right\}\right)=90$ (the inclusion of $\Gamma_{4}$ triggers the inclusion of $\left.\Gamma_{7}\right), R\left(\left\{\Gamma_{2}, \Gamma_{3}\right\}\right)=120, R\left(\left\{\Gamma_{2}, \Gamma_{3}, \Gamma_{7}\right\}\right)=140$, $R\left(\left\{\Gamma_{3}, \Gamma_{4}, \Gamma_{7}\right\}\right)=140,\left(\left\{\Gamma_{1}\right\}\right)=150$, and so on. The total number of possible subsets according to the graph $\mathcal{G}$ in Fig. 7 is 16.

This approach, while finding the optimal subset $\Omega$, requires a large amount of computation time and can be used only with a small number of applications.

2) Greedy Heuristic (GH): If the number of applications is larger, a possible solution could be based on a simple greedy heuristic which, starting from $\Omega=\varnothing$, progressively enlarges the subset until a valid solution is produced. The algorithm looks at all the nonfrozen applications and picks that one which, together with its dependencies, has the smallest modification cost. If the new subset does not produce a valid solution, it is enlarged by including, in the same fashion, the next application with its dependencies. This greedy expansion of the subset is continued until the set is large enough to lead to a valid solution or no application is left. For the example in Fig. 7 the call to NextSubset $(\varnothing)$ will produce $R\left(\left\{\Gamma_{7}\right\}\right)=20$, and will be suc- 


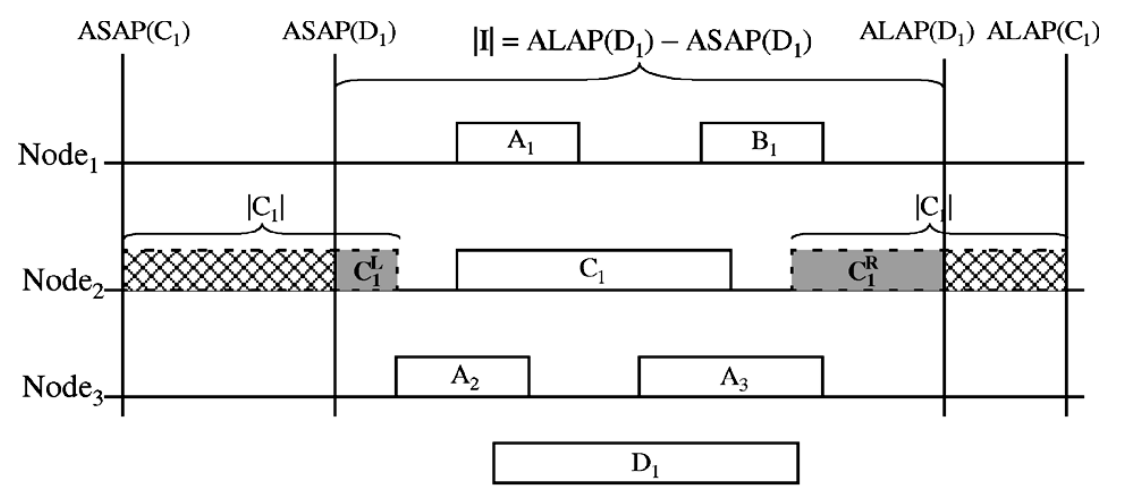

$$
\begin{aligned}
& \delta_{B}^{D_{1}}=\max \left(|I|-\left|A_{1}\right|-\min \left(\left|B_{1}^{L}\right|,\left|B_{1}^{R}\right|\right)-\left|D_{1}\right|, 0\right) ; \delta_{C}^{D_{1}}=\max \left(|I|-\min \left(\left|\mathrm{C}_{1}^{L}\right|,\left|C_{1}^{R}\right|\right)-\left|D_{1}\right|, 0\right) \\
& \delta_{A}^{D_{1}}=\max \left(\operatorname { m a x } \left(|I|-\left|B_{1}\right|-\min \left(\left|A_{1}^{L}\right|,\left|A_{1}^{R}\right|\right), \frac{\left.\left.I I-\min \left(\left|A_{2}^{L}\right|,\left|A_{2}^{R}\right|\right)-\min \left(\left|A_{3}^{L}\right|,\left|A_{3}^{R}\right|\right)\right)-\left|D_{1}\right|, 0\right)}{D_{1} \text { mapped on Node }}\right.\right.
\end{aligned}
$$

Fig. 13. Metric for the subset selection heuristic.

cessively enlarged to $R\left(\left\{\Gamma_{7}, \Gamma_{3}\right\}\right)=70, R\left(\left\{\Gamma_{7}, \Gamma_{3}, \Gamma_{2}\right\}\right)=$ $140\left(\Gamma_{40}\right.$ could have been picked as well in this step because it has the same modification cost of 70 as $\Gamma_{2}$ and its dependence $\Gamma_{7}$ is already in the subset), $R\left(\left\{\Gamma_{7}, \Gamma_{3}, \Gamma_{2}, \Gamma_{4}\right\}\right)=210$, and so on.

While this approach finds very quickly a valid solution, if one exists, it is possible that the resulted total modification cost is much higher than the optimal one.

3) Subset Selection Heuristic (SH): An intelligent selection heuristic should be able to identify the reasons due to which a valid solution has not been produced and to find the set of candidate applications which, if modified, could eliminate the problem. The failure to produce a valid solution can have two possible causes: an initial mapping which meets the deadlines has not been found, or the second criterion is not satisfied.

Let us investigate the first reason. If an application $\Gamma_{i}$ is to meet its deadline $D_{i}$, all its processes $P_{j} \in \Gamma_{i}$ have to be scheduled inside their [ASAP, ALAP] intervals. InitialMappingScheduling (IMS) fails to schedule a process inside its [ASAP, ALAP] interval if there is not enough slack available on any processor, due to other processes scheduled in the same interval. In this situation we say that there is a conflict with processes belonging to other applications. We are interested to find out which applications are responsible for conflicts encountered during the mapping and scheduling of $\Gamma_{\text {current }}$, and not only that, but also which ones are flexible enough to be moved away in order to avoid these conflicts.

If it is not able to find a solution that satisfies the deadlines, IMS will determine a metric $\Delta_{\Gamma_{i}}$ that characterizes both the degree of conflict and the flexibility of each application $\Gamma_{i} \in \Psi$ in relation to $\Gamma_{\text {current }}$. A set of applications $\Omega$ will be characterized, in relation to $\Gamma_{\text {current }}$, by the following metric:

$$
\Delta(\Omega)=\sum_{\Gamma_{i} \in \Omega} \Delta_{\Gamma_{i}}
$$

This metric $\Delta(\Omega)$ will be used by our subset selection heuristic in the case IMS has failed to produce a solution which satisfies the deadlines. An application with a larger $\Delta_{\Gamma_{i}}$ is more likely to lead to a valid schedule if included in $\Omega$.
In Fig. 13, we illustrate how this metric is calculated. Applications $A, B$ and $C$ are implemented on a system consisting of the three processors Node 1, Node $_{2}$ and Node 3 . The current application to be implemented is $D$. At a certain moment, IMS comes to the point to map and schedule process $D_{1} \in D$. However, it is not able to place it inside its [ASAP, ALAP] interval, denoted in Fig. 13 as $I$. The reason is that there is not enough slack available inside $I$ on any of the processors, because processes $A_{1}, A_{2}, A_{3} \in A, B_{1} \in B$, and $\mathrm{C}_{1} \in C$ are scheduled inside that interval. We are interested to determine which of the applications $A, B$, and $C$ are more likely to lend free slack for $D_{1}$, if remapped and rescheduled. Therefore, we calculate the slack resulted after we move away processes belonging to these applications from the interval $I$. For example, the resulted slack available after modifying application $C$ (moving $\mathrm{C}_{1}$ either to the left or to the right inside its own [ASAP, ALAP] interval) is of size $|I|-\min \left(\left|\mathrm{C}_{1}^{L}\right|,\left|\mathrm{C}_{1}^{R}\right|\right)$. With $\mathrm{C}_{1}^{L}\left(\mathrm{C}_{1}^{R}\right)$ we denote that slice of process $\mathrm{C}_{1}$ which remains inside the interval $I$ after $\mathrm{C}_{1}$ has been moved to the extreme left (right) inside its own [ASAP, ALAP] interval. $\left|\mathrm{C}_{1}^{L}\right|$ represents the length of slice $\mathrm{C}_{1}^{L}$. Thus, when considering process $D_{1}, \Delta_{C}$ will be incremented with $\delta_{C}^{D_{1}}=\max \left(|I|-\min \left(\left|\mathrm{C}_{1}^{L}\right|,\left|\mathrm{C}_{1}^{R}\right|\right)-\left|D_{1}\right|, 0\right)$. This value shows the maximum theoretical slack usable for $D_{1}$, that can be produced by modifying application $C$. By relating this slack to the length of $D_{1}$, the value $\delta_{C}^{D_{1}}$ also captures the amount of flexibility provided by that modification.

The increments $\delta_{B}^{D_{1}}$ and $\delta_{A}^{D_{1}}$ to be added to the values of $\Delta_{B}$ and $\Delta_{A}$ respectively, are also presented in Fig. 13. IMS then continues the evaluation of the metrics $\Delta$ with the other processes belonging to the current application $\mathrm{D}$ (with the assumption that process $D_{1}$ has been scheduled at the beginning of interval $I$ ). Thus, as result of the failed attempt to map and schedule application $D$, the metrics $\Delta_{A}, \Delta_{B}$, and $\Delta_{C}$ will be produced.

If the initial mapping was successful, the first step of MS could fail during the attempt to satisfy the second criterion (Fig. 11). In this case, the metric $\Delta_{\Gamma_{i}}$ is computed in a different way. What $\Delta_{\Gamma_{i}}$ will capture in this case, is the potential of an application $\Gamma_{i}$ to improve the metric $\mathrm{C}_{2}$ if remapped 
together with $\Gamma_{\text {current }}$. Therefore, we consider a total number of moves from all the nonfrozen applications. These moves are determined using the PotentialMove $\mathrm{C}_{2}$ functions presented in Section VI-B. Each such move will lead to a different mapping and schedule, and thus to a different $\mathrm{C}_{2}$ value. Let us consider $\delta_{\text {move }}$ as the improvement on $\mathrm{C}_{2}$ produced by the currently considered move. If there is no improvement, $\delta_{\text {move }}=0$. Thus, for each move that has as subject $P_{j}$ or $m_{j} \in \Gamma_{i}$, we increment the metric $\Delta_{\Gamma_{i}}$ with the $\delta_{\text {move }}$ improvement on $\mathrm{C}_{2}$.

As shown in the algorithm in Fig. 11, MS starts by trying an implementation of $\Gamma_{\text {current }}$ with $\Omega=\varnothing$. If this attempt fails, because of one of the two reasons mentioned above, the corresponding metrics $\Delta_{\Gamma_{i}}$ are computed for all $\Gamma_{i} \in \Psi$. Our heuristic SH will then start by finding the solution $\Omega_{\mathrm{GH}}$ produced with the greedy heuristic GH (this will succeed if there exists any solution). The total modification cost corresponding to this solution is $R_{\mathrm{GH}}=R\left(\Omega_{\mathrm{GH}}\right)$ and the value of the metric $\Delta$ is $\Delta_{\mathrm{GH}}=\Delta\left(\Omega_{\mathrm{GH}}\right)$. SH now continues by trying to find a solution with a more favorable $\Omega$ than $\Omega_{\mathrm{GH}}$ (a smaller total cost $R$ ). Therefore, the thresholds $R_{\max }=R_{\mathrm{GH}}$ and $\Delta_{\min }=\Delta_{\mathrm{GH}} / n$ (for our experiments we considered $n=2$ ) are set. Sets of applications not fulfilling these thresholds will not be investigated by MS. For generating new subsets $\Omega$, the function NextSubset now follows a similar approach like in the exhaustive search approach ES, but in a reverse direction, toward smaller subsets (starting with the set containing all nonfrozen applications), and it will consider only subsets with a smaller total cost then $R_{\max }$ and a larger $\Delta$ than $\Delta_{\min }$ (a small $\Delta$ means a reduced potential to eliminate the cause of the initial failure). Each time a valid solution is found, the current values of $R_{\max }$ and $\Delta_{\min }$ are updated in order to further restrict the search space. The heuristic stops when no subset can be found with $\Delta>\Delta_{\min }$, or a certain imposed limit has been reached (e.g., on the total number of attempts to find new subsets).

\section{EXPERIMENTAL RESULTS}

In Section VII-A-C, we show a series of experiments that demonstrate the effectiveness of the proposed approach and algorithms. The first set of results is related to the efficiency of our mapping and scheduling algorithm and the iterative design transformations proposed in Sections VI-A and B. The second set of experiments evaluates our heuristics for minimization of the total modification cost presented in Section VI-C. As a general strategy, we have evaluated our algorithms performing experiments on a large number of test cases generated for experimental purpose. Finally, we have validated the proposed approach using a real-life example. All experiments were run on a SUN Ultra 10 workstation.

\section{A. Evaluation of the IMS Algorithm and the Iterative Design Transformations}

For an evaluation of our approach we used process graphs of $80,160,240,320$, and 400 processes, representing the application $\Gamma_{\text {current }}$, randomly generated for experimental purpose. Thirty graphs were generated for each graph dimension, thus a total of 150 graphs were used for experimental evaluation.
TABLE I

EVALUATION OF THE INITIAL MAPPING AND SCHEDULING

\begin{tabular}{c||c|c|c|c|c|c}
\hline \multicolumn{1}{l||}{$\begin{array}{l}\text { No. of } \\
\text { Procs. }\end{array}$} & \multicolumn{3}{c|}{ HCP } & \multicolumn{3}{c}{ IMS } \\
\cline { 2 - 7 } & avg. & max. & better & avg. & max. & better \\
\hline \hline 80 & $2.04 \%$ & $31.57 \%$ & $10 \%$ & $0.35 \%$ & $1.47 \%$ & $30 \%$ \\
\hline 160 & $3.12 \%$ & $48.89 \%$ & $10 \%$ & $1.18 \%$ & $5.44 \%$ & $33.33 \%$ \\
\hline 240 & $5.53 \%$ & $61.27 \%$ & $13.33 \%$ & $1.38 \%$ & $14.52 \%$ & $36.66 \%$ \\
\hline 320 & $6.12 \%$ & $88.57 \%$ & $16.66 \%$ & $2.79 \%$ & $24.33 \%$ & $40 \%$ \\
\hline 400 & $11.02 \%$ & $120.77 \%$ & $13.33 \%$ & $2.78 \%$ & $22.52 \%$ & $36.66 \%$ \\
\hline
\end{tabular}

We generated both graphs with random structure and graphs based on more regular structures like trees and groups of chains. We generated a random structure graph deciding for each pair of two processes if they should be connected or not. Two processes in the graph were connected with a certain probability (between 0.05 and 0.15 , depending on the graph dimension) on the condition that the dependency would not introduce a loop in the graph. The width of the tree-like structures was controlled by the maximum number of direct successors a process can have in the tree (from 2 to 6), while the graphs consisting of groups of chains had 2 to 12 parallel chains of processes. Furthermore, the regular structures were modified by adding a number of 3 to 30 random cross-connections.

Execution times and message lengths were assigned randomly using both uniform and exponential distribution within the 10 to $100 \mathrm{~ms}$, and 2 to 8 bytes ranges, respectively.

We considered an architecture consisting of 10 nodes of different speeds. For the communication channel we considered a transmission speed of $256 \mathrm{kBps}$ and a length below $20 \mathrm{~m}$. The maximum length of the data field in a bus slot was 8 bytes.

Throughout the experiments presented in this section we have considered an existing set of applications $\Psi$ consisting of 400 processes, with a schedule table of $6 \mathrm{~s}$ on each processor, and a slack of about $50 \%$ of the total schedule size. The mapping of the existing applications has been done using a simple heuristic that tries to balance the utilization of processors while minimizing communication. The scheduling of the applications $\Psi$ has been performed using list scheduling, and the schedules obtained have then been stretched to their deadline by introducing slacks distributed uniformly over the schedule table.

In this section, we have also considered that no modifications of the existing set of applications $\Psi$ are allowed when implementing a new application. We will concentrate on the aspects related to the modification of existing applications, in the following section.Our heuristic (MS), proposed in VI-B, as well as SA and AH have been used to map and schedule each of the 150 process graphs on the target system. For each of the resulted designs, the objective function $C$ has been computed. Very long and expensive runs have been performed with the SA algorithm for each graph and the best ever solution produced has been considered as the near optimum for that graph. We have compared the objective function obtained for the 150 process graphs considering each of the three heuristics. Fig. 14(a) presents the average percentage deviation of the objective function obtained with the MS and AH from the value of the objective function obtained with the near-optimal scheme (SA). We have excluded 


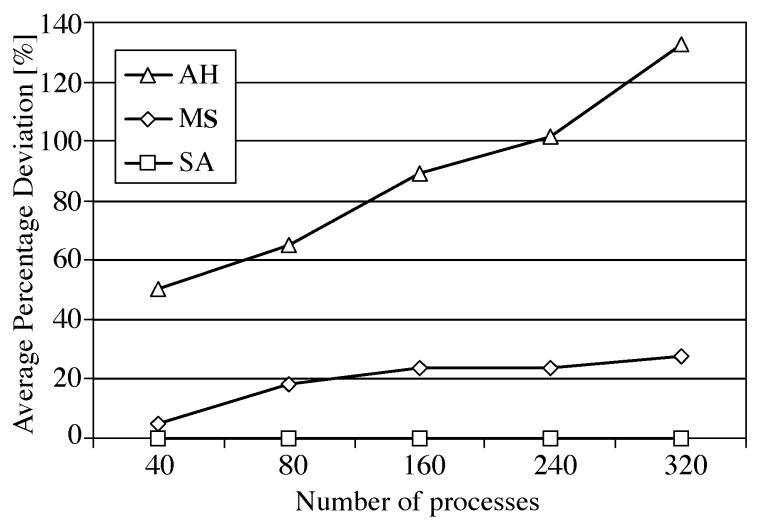

(a)

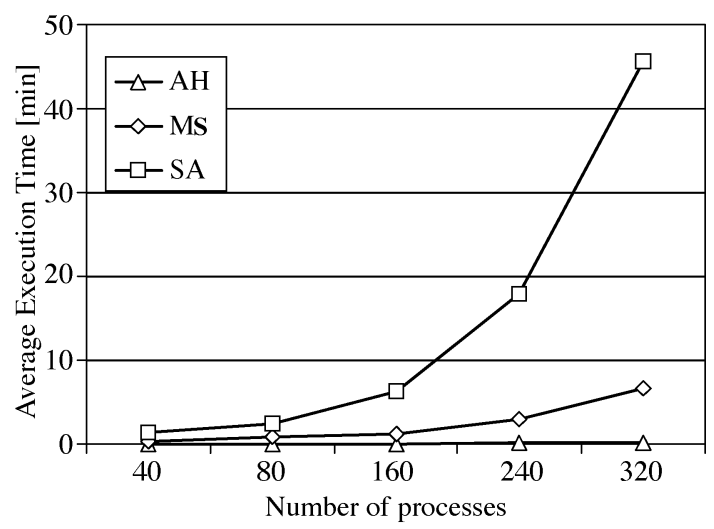

(b)

Fig. 14. Evaluation of the design transformation heuristics: a) deviation of the objective function obtained with MS and AH from that obtained with SA and b) execution times.

from the results in Fig. 14(a), 37 solutions obtained with AH for which the second design criterion has not been met, and thus the objective function has been strongly penalized. The average run-times of the algorithms are presented in Fig. 14(b). The SA approach performs best in terms of quality at the expense of a large execution time: The execution time can be up to $45 \mathrm{~min}$ for large graphs of 400 processes. The important aspect is that MS performs very well, and is able to obtain good quality solutions, very close to those produced with SA, in a very short time. AH is, of course, very fast, but since it does not address explicitly the two design criteria presented in Section V it has the worst quality of solutions, as expressed by the objective function.

The first result concerns the quality of the designs produced by our initial mapping and scheduling algorithm IMS. As discussed in Section VI-A, IMS uses the MPCP priority function which considers particularities of the TDMA protocol. In our experiments we compared the quality of designs (in terms of schedule length) produced by IMS with those generated with the original HCP algorithm proposed in [13]. Results are depicted in Table I where we have three columns for both HCP and IMS. In the columns labeled "average" we present the average percentage deviations of the schedule length produced with HCP and IMS from the length of the best schedule among the two. In the "maximum" column we have the maximum percentage deviation, and the column with the heading "better" shows the percentage of cases in which HCP or IMS was better than the other. For example, for 240 processes, HCP had an average percentage deviation from the best result of $5.53 \%$, compared to $1.38 \%$ for IMS. Also, in the worst case, the schedule length obtained with HCP was $61.27 \%$ larger than the one obtained with IMS. There were four cases (13.33\%) in which HCP has obtained a better result than IMS, compared to 11 cases $(36.66 \%)$ where IMS has obtained a better result. For the rest of the 15 cases, the schedule lengths obtained were equal. We can observe that, inaverage, the deviationfrom the bestresultis 3.28 times smaller with IMS than with HCP. The average execution times for both algorithms are under half a second for graphs with 400 processes.

For the next set of experiments we were interested to investigate the quality of the design transformation heuristic discussed in Section VI-B, aiming at the optimization of the objective function $C$. In order to compare this heuristic, implemented in our mapping

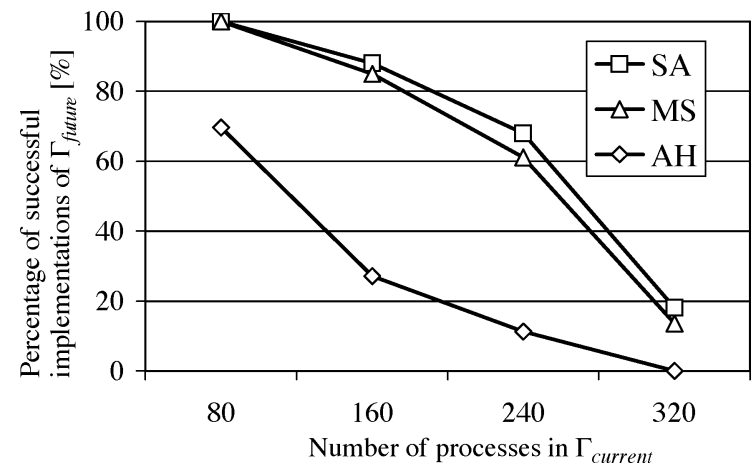

Fig. 15. Percentage of future applications successfully implemented.

and scheduling approach MS, we have developed two additional heuristics:

1) Asimulatedannealing strategy (SA) [36], based on the same moves as described in Section VI-B. SA is applied on the solution produced by IMS and aims at finding the near-optimal mapping and schedule that minimizes the objective function $C$. The main drawback of the SA strategy is that in order to find the nearoptimal solution it needs very large computation times. Such a strategy, although useful for the final stages of the system synthesis, cannot be used inside a design space exploration cycle.

2) A so called ad hoc approach (AH) which is a simple, straight-forward solution to produce designs that, to a certain degree, support an incremental process. Starting from the initial valid schedule of length $S$ obtained by IMS for a graph $G$ with $N$ processes, AH uses a simple scheme to redistribute the processes inside the $[0, D]$ interval, where $D$ is the deadline of process graph $G$. AH starts by considering the first process in topological order, let it be $P_{1}$. It introduces after $P_{1}$ a slack of size max (smallest process size of $\left.\Gamma_{\text {future }},(D-S) / N\right)$, thus shifting all descendants of $P_{1}$ to the right (toward the end of the schedule table). The insertion of slacks is repeated for the next process, with the current, larger value of $S$, as long as the resulted schedule has a length $S \leq D$. Processes are moved only as long as their individual deadlines (if any) are not violated.

The most important aspect of the experiments is determining to which extent the design transformations proposed by us, and 


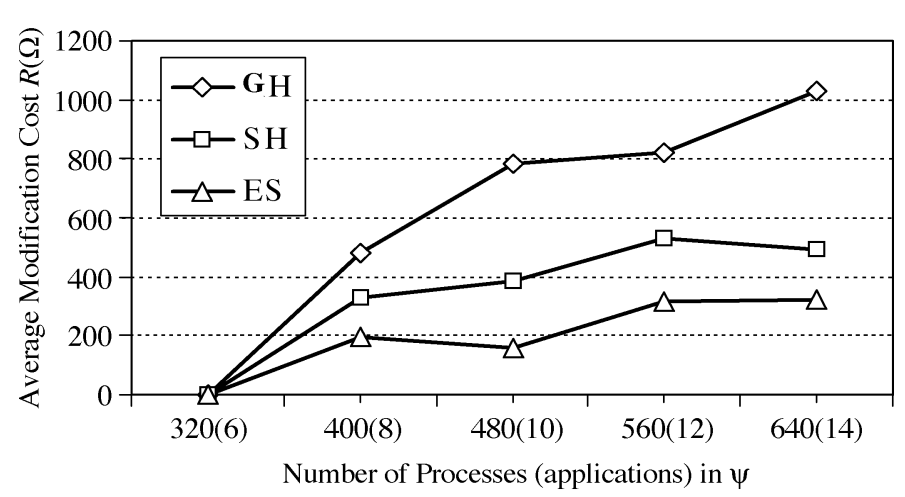

(a)

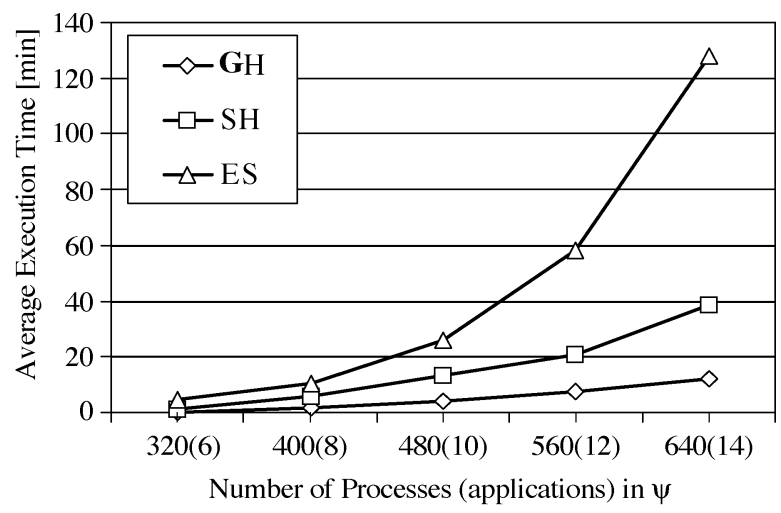

(b)

Fig. 16. Evaluation of the modification cost minimization. a) Modification cost obtained with the GH, SH, and ES heuristics and b) execution times.

the related heuristic, really facilitate the implementation of future applications. To find this out, we have mapped graphs of 80 , 160,240 , and 320 nodes representing the $\Gamma_{\text {current }}$ application on top of $\Psi$ (the same $\Psi$ as defined for the previous set of experiments). After mapping and scheduling each of these graphs we have tried to add a new application $\Gamma_{\text {future }}$ to the resulted system. $\Gamma_{\text {future }}$ consists of a process graph of 80 processes, randomly generated according to the following specifications: $S_{t}=$ $\{20,50,100,150,200 \mathrm{~ms}\}, f_{t}\left(S_{t}\right)=\{10,25,45,15,5 \%\}$, $S_{b}=\{2,4,6,8$ bytes $\}, f_{b}\left(S_{b}\right)=\{20,50,20,10 \%\}, T_{\min }=$ $250 \mathrm{~ms}, t_{\text {need }}=100$ and $b_{\text {need }}=20 \mathrm{~ms}$. The experiments have been performed three times: using MS, SA, and AH for mapping $\Gamma_{\text {current }}$. In all three cases, we were interested if it is possible to find a correct implementation for $\Gamma_{\text {future }}$ on top of $\Gamma_{\text {current }}$ using the initial mapping and scheduling algorithm IMS (without any modification of $\Psi$ or $\Gamma_{\text {current }}$ ). Fig. 15 shows the percentage of successful implementations of $\Gamma_{\text {future }}$ for each the three cases. In the case $\Gamma_{\text {current }}$ has been implemented with MS and SA, this means using the design criteria and metrics proposed in the paper, we were able to find a valid schedule for $65 \%$ and $68 \%$ of the total cases, respectively. However, using AH to map $\Gamma_{\text {current }}$, has led to a situation where IMS is able to find correct solutions in only $21 \%$ of the cases. Another conclusion from Fig. 15 is that when the total slack available is large, as in the case $\Gamma_{\text {current }}$ has only 80 processes, it is easy for MS and, to a certain extent, even for $\mathrm{AH}$ to find a mapping that allows adding future applications. However, as $\Gamma_{\text {current }}$ grows to 240 processes, only MS and SA are able to find an implementation of $\Gamma_{\text {current }}$ that supports an incremental design process, accommodating the future application in more than $60 \%$ of the cases. If the remaining slack is very small, after we map a $\Gamma_{\text {current }}$ of 320 processes, it becomes practically impossible to map new applications without modifying the current system. Moreover, our mapping heuristic $\mathrm{MH}$ performs very well compared to the simulated annealing approach SA which aims for the near-optimal value of the objective function.

\section{B. Evaluation of the Modification Cost Minimization Heuristics}

For this set of experiments we first used the same 150 process graphs as in the previous section, consisting of $80,160,240$,
320, and 400 processes, for the application $\Gamma_{\text {current }}$. We also considered the same system architecture as presented there.

The first results concern the quality of the solution obtained with our mapping strategy MS using the search heuristic SH compared to the case when the simple greedy approach GH and the exhaustive search ES are used. For the existing applications we have generated five different sets $\Psi$, consisting of different numbers of applications and processes, as follows: 6 applications (320 processes), 8 applications (400 processes), 10 applications (480 processes), 12 applications (560 processes), and 14 applications (640 processes). The process graphs in the applications as well as their mapping and scheduling were generated as described in the introduction of Section VII-A.

After generating the applications we have manually assigned modification costs in the range 10 to 100 , depending on their size. The dependencies between applications (in the sense introduced in Section IV-A) were such that the total number of possible subsets $\Omega$ resulted for each set $\Psi$ were 32, 128, 256, 1024, and 4096, respectively. We have considered that the future applications, $\Gamma_{\text {future }}$, are characterized by the following parameters: $S_{t}=\{20,50,100,150,200 \mathrm{~ms}\}$, $f_{t}\left(S_{t}\right)=\{10,25,45,15,5 \%\}, S_{b}=\{2,4,6,8$ bytes $\}$, $f_{b}\left(S_{b}\right)=\{20,50,20,10 \%\}, T_{\min }=250 \mathrm{~ms}, t_{\text {need }}=100 \mathrm{~ms}$ and $b_{\text {need }}=20 \mathrm{~ms}$.

MS has been used to produce a valid solution for each of the 150 process graphs representing $\Gamma_{\text {current }}$, on each of the target configurations $\Psi$, using the $\mathrm{ES}, \mathrm{GH}$, and $\mathrm{SH}$ approaches to subset selection. Fig. 16(a) compares the three approaches based on the total modification costneededin ordertoobtain a valid solution. Theexhaustive approach ES is able to obtain valid solutions with an optimal (smallest) modification cost, while the greedy approach GH produces in average 3.12 times more costly modifications in order to obtain valid solutions. However, in order to find the optimal solution, ES needs large computation times, as shown in Fig. 16(b). For example, it can take more than an average of $2 \mathrm{~h}$ to find the smallest cost subset to be remapped that leads to a valid solution in the case of 14 applications ( 640 processes). We can see that the proposed heuristic SH performs well, producing close to optimal results with a good scaling for large application sets. For the results in Fig. 16, we have eliminated those situations in which novalid solution could be produced by MS.

Finally, we have repeated the last set of experiments discussed in the previous section (the experiments leading to the results 
in Fig. 15). However, in this case, we have allowed the current system (consisting of $\Psi \cup \Gamma_{\text {current }}$ ) to be modified when implementing $\Gamma_{\text {future. }}$ If the mapping and scheduling heuristic is allowed to modify the existing system then we are able to increase the total number of successful attempts to implement application $\Gamma_{\text {future }}$ from $65 \%$ to $77.5 \%$. For the case with $\Gamma_{\text {future }}$ consisting of 160 processes (when the amount of available resources for $\Gamma_{\text {future }}$ is small) the increase is from $60 \%$ to $92 \%$. Such an increase is, of course, expected. The important aspect, however, is that it is obtained not by randomly selecting old applications to be modified, but by performing this selection such that the total modification cost is minimized.

\section{The Vehicle Cruise Controller}

A typical safety critical application with hard real-time constraints, to be implemented on a TTP based architecture, is a vehicle cruise controller (CC). We have considered a $\mathrm{CC}$ system derived from a requirement specification provided by the industry. The $\mathrm{CC}$ delivers the following functionality: it maintains a constant speed for speeds over $35 \mathrm{~km} / \mathrm{h}$ and under $200 \mathrm{~km} / \mathrm{h}$, offers an interface (buttons) to increase or decrease the reference speed, and is able to resume its operation at the previous reference speed. The CC operation is suspended when the driver presses the brake pedal. The specification assumes that the $\mathrm{CC}$ will operate in an environment consisting of several nodes interconnected by a TTP channel (Fig. 17). There are four nodes which functionally interact with the $\mathrm{CC}$ system: the anti-lock braking system (ABS), the transmission control module (TCM), the engine control module (ECM), and the electronic throttle module (ETM). It has been decided to map the functionality (processes) of the CC over these four nodes. The ECM and ETM nodes have an 8-bit Motorola M68HC11 family CPU with 128 $\mathrm{kB}$ of memory, while the ABS and TCM are equipped with a 16-bit Motorola M68HC12 CPU and $256 \mathrm{kB}$ of memory. The 16-bit CPUs are twice faster than the 8-bit ones. The transmission speed of the communication channel is $256 \mathrm{~kb} / \mathrm{s}$ and the frequency of the TTP controller was chosen to be $20 \mathrm{MHz}$. We have modeled the specification of the CC system using a set of 32 processes and 17 messages as described in [28]. The period was $300 \mathrm{~ms}$, equal to the deadline.

The system $\Psi$, representing the applications already running on the four nodes mentioned earlier, has been modeled as a set of 80 processes with a schedule table of $300 \mathrm{~ms}$ and leaving a total of $40 \%$ slack. We have assigned to each application a modification cost proportional to the number and size of processes. The $\mathrm{CC}$ is the $\Gamma_{\text {current }}$ application to be implemented. We have also generated 30 future applications of 40 processes each, with the general characteristics close to those of the $\mathrm{CC}$, which are typical for automotive applications. We have first mapped and scheduled the CC on top of $\Psi$, using the ad-hoc strategy (AH) and then our MS algorithm. On the resulted systems, consisting of $\Psi \cup \mathrm{CC}$, we tried to implement each of the 30 future applications. First we considered a situation in which no modifications of the existing system are allowed when implementing the future applications. In this case, we were able to implement 21 of the 30 future applications after implementing the CC with MS, while using $\mathrm{AH}$ to implement the $\mathrm{CC}$, only 4 of the future ap-

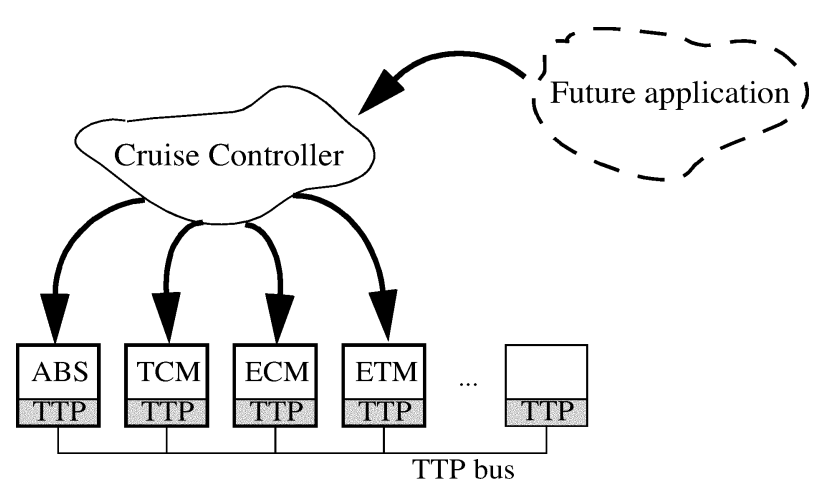

Fig. 17. Implementation of the cruise controller.

plications could be mapped. When modifications of the current system were allowed, using MS, we were able to map 24 of the 30 future applications on top of the CC. For the CC example SA has obtained the same results as MS.

\section{CONCLUSIONS}

We have presented an approach to the incremental design of distributed hard real-time embedded systems. Such a design process satisfies two main requirements when adding new functionality: already running applications are disturbed as little as possible, and there is a good chance that, later, new functionality can easily be mapped on the resulted system. Our approach assumes a nonpreemptive static cyclic scheduling policy and a realistic communication model based on a TDMA scheme.

We have introduced two design criteria with their corresponding metrics that drive our mapping strategy to solutions supporting an incremental design process. These solutions are obtained using an efficient transformation based heuristic.

Three algorithms have been proposed to produce a minimal subset of applications which have to be remapped and rescheduled in order to implement the new functionality. ES is based on a, potentially slow, branch and bound strategy which finds an optimal solution. GH is very fast but produces solutions that could be of too high cost, while $\mathrm{SH}$ is able to quickly produce good quality results.

The approach has been evaluated based on extensive experiments using a large number of generated benchmarks as well as a real-life example.

Although the concrete architecture used to illustrate our approach is a distributed embedded system, typically used in automotive applications, the proposed strategy and heuristics can as well be used for on-chip architectures and platforms.

There are several aspects that have been omitted from the discussion in this paper. In [29], we have extended our approach to real-time systems where process scheduling is based on a static priority preemptive approach. For the sake of simplifying the discussion, we have also not addressed here the memory constraints during process mapping and the implications of memory space in the incremental design process. An extension of the approach in order to consider memory space as another resource in addition to processor time and bus bandwidth is, however, straightforward. We have also not discussed in this paper the issue of architecture selection, considering that the designer has taken the appropriate decisions before starting the mapping and scheduling procedure. 


\section{REFERENCES}

[1] J. E. Beck and D. P. Siewiorek, "Automatic configuration of embedded multicomputer systems," IEEE Trans. Computer-Aided Design, vol. 17, pp. 84-95, Feb. 1998.

[2] T. Blicke, J. Teich, and L. Thiele, "System-level synthesis using evolutionary algorithms," Des. Autom. Embedded Syst., vol. 4, no. 1, pp. 23-58, 1998.

[3] B. W. Boehm et al., Software Cost Estimation With COCOMO II. Englewood Cliffs, NJ: Prentice-Hall, 2000.

[4] E. G. Coffman Jr. and R. L. Graham, "Optimal scheduling for two processor systems," Acta Informatica, no. 1, pp. 200-213, 1972.

[5] B. P. Dave, G. Lakshminarayana, and N. K. Jha, "COSYN: hardwaresoftware co-synthesis of heterogeneous distributed embedded systems," IEEE Trans. VLSI Syst., vol. 7, pp. 92-104, Mar. 1999.

[6] J. A. Debardelaben, V. K. Madiseti, and A. J. Gadient, "Incorporating cost modeling in embedded-system design," IEEE Des. Test Comput., pp. 24-35, July-Sept. 1997.

[7] P. Eles, Z. Peng, K. Kuchcinski, and A. Doboli, "System level hardware/software partitioning based on simulated annealing and tabu search," Des. Autom. Embedded Syst., vol. 2, no. 1, pp. 5-32, 1997.

[8] P. Eles, A. Doboli, P. Pop, and Z. Peng, "Scheduling with bus access optimization for distributed embedded systems," IEEE Trans. VLSI Syst. vol. 8, pp. 472-491, Oct. 2000.

[9] R. Ernst, J. Henkel, and T. Benner, "Hardware-software cosynthesis for microcontrollers," IEEE Des. Test Comput., vol. 10, pp. 64-75, Sept. 1993.

[10] R. Ernst, "Codesign of embedded systems: status and trends," IEEE Des. Test Comput., pp. 45-54, Apr.-June 1998.

[11] D. D. Gajski and F. Vahid, "Specification and design of embedded hardware-software systems," IEEE Des. Test Comput., pp. 53-67, Spring 1995.

[12] R. K. Gupta and G. De Micheli, "Hardware-software cosynthesis for digital systems," IEEE Des. Test Comput., vol. 10, pp. 29-41, Sept. 1993.

[13] P. B. Jorgensen and J. Madsen, "Critical path driven cosynthesis for heterogeneous target architectures," in Proc. Int. Workshop on Hardware-Software Co-Design, 1997, pp. 15-19.

[14] C. Haubelt, J. Teich, K. Richter, and R. Ernst, "System design for flexibility," in Proc. Design, Automation and Test Eur. Conf., 2002, pp. 854-861.

[15] A. Kalawade and E. A. Lee, "The extended partitioning problem: hardware/software mapping, scheduling, and implementation-bin selection," Des. Autom. Embedded Syst., vol. 2, pp. 125-163, 1997.

[16] P. V. Knudsen and J. Madsen, "Integrating communication protocol selection with hardware/software codesign," IEEE Trans. Computer-Aided Design, vol. 18, no. 8, pp. 1077-1095, 1999.

[17] H. Kopetz, Real-Time Systems-Design Principles for Distributed Embedded Applications. $\quad$ Norwell, MA: Kluwer, 1997.

[18] H. Kopetz and G. Grünsteidl, "TTP-a protocol for fault-tolerant realtime systems," IEEE Comput., vol. 27, pp. 14-23, Jan. 1994.

[19] K. Keutzer, S. Malik, R. Newton, J. Rabaey, and A. Sangiovanni-Vincentelli, "System-level design: orthogonalization of concerns and platform-based design," IEEE Trans. Computer-Aided Design, vol. 19, no. 12 , pp. $1523-1543$, Dec. 2000

[20] C. Lee, M. Potkonjak, and W. Wolf, "Synthesis of hard real-time application specific systems," Des. Autom. Embedded Syst., vol. 4, no. 4, pp. 215-241, 1999.

[21] Y. Li, T. Callahan, E. Darnell, R. Harr, U. Kurkure, and J. Stockwood, "Hardware-software co-design of embedded reconfigurable architectures," in Proc. Design Automation Conf., 2000, pp. 507-512.

[22] S. Martello and P. Toth, Kanpsack Problems: Algorithms and Computer Implementations. New York: Wiley, 1990.

[23] G. Martin and F. Schirrmeister, "A design chain for embedded systems," Computer, vol. 35, no. 3, pp. 100-103, Mar. 2002.

[24] G. De Micheli and M. G. Sami, Eds., Hardware/Software Co-Design. Norwell, MA: Kluwer, 1996.

[25] G. De Micheli and R. K. Gupta, "Hardware/software co-design," Proc. IEEE, vol. 85, pp. 349-365, 1997.

[26] S. Narayan and D. D. Gajski, "Synthesis of system-level bus interfaces," in Proc. Eur. Desing and Test Conf., 1994, pp. 395-399.

[27] R. B. Ortega and G. Borriello, "Communication synthesis for distributed embedded systems," in Proc. Int. Conf. CAD, 1998, pp. 437-444.

[28] P. Pop, "Analysis and Synthesis of Communication-Intensive Heterogeneous Real-Time Systems," Ph.D. dissertation, Linköping Studies in Science and Technology, Linköping, Sweden.

[29] P. Pop, P. Eles, and Z. Peng, "Flexibility driven scheduling and mapping for distributed real-time systems," in Proc. Int. Conf. Real-Time Computing Systems and Applications, 2002, pp. 337-346.
[30] _ - "Scheduling with optimized communication for time-triggered embedded systems," in Proc. Int. Workshop on Hardware-Software Co-Design, 1999, pp. 178-182.

[31] P. Pop, P. Eles, T. Pop, and Z. Peng, "An approach to incremental design of distributed embedded systems," in Proc. Design Automation Conf., 2001, pp. 450-455.

[32] - "Minimizing system modification in an incremental design approach," in Proc. Int. Workshop on Hardware/Software Codesign, 2001, pp. $183-188$.

[33] S. Prakash and A. Parker, "SOS: synthesis of application-specific heterogeneous multiprocessor systems," J. Parallel Distrib. Comput., vol. V16, pp. 338-351, 1992.

[34] P. Puschner and A. Burns, "A review of worst-case execution-time analyzes," Real-Time Syst. J., vol. 18, no. 2/3, pp. 115-128, May 2000.

[35] D. Ragan, P. Sandborn, and P. Stoaks, "A detailed cost model for concurrent use with hardware/software co-design," in Proc. Design Automation Conf., 2002, pp. 269-274.

[36] C. R. Reevs, Modern Heuristic Techniques for Combinatorial Problems. Oxford, U.K: Blackwell, 1993.

[37] D. L. Rhodes and W. Wolf, "Co-synthesis of heterogeneous multiprocessor systems using arbitrated communication," in Proc. Int. Conf. $C A D, 1999$, pp. 339-342.

[38] A. Sangiovanni-Vincentelli, "Electronic-system design in the automobile industry," IEEE Micro, vol. 23, pp. 8-18, May-June 2003.

[39] J. Staunstrup and W. Wolf, Eds., Hardware/Software Co-Design: Principles and Practice. Norwell, MA: Kluwer, 1997.

[40] W. Wolf, "An architectural co-synthesis algorithm for distributed, embedded computing systems," IEEE Trans. VLSI Syst., pp. 218-229, June 1997.

[41] T. Y. Yen and W. Wolf, Hardware-Software Co-Synthesis of Distributed Embedded Systems. Norwell, MA: Kluwer, 1997.

[42] W. Wolf, "Hardware-software co-design of embedded systems," Proc. IEEE, vol. 82, no. 7, pp. 967-989, 1994.

[43] REVIC Software Cost Estimating Model, User's Manual, V9.0-9.2, U.S. Air Force Analysis Agency, 1994.

[44] SoftEST-Version 1.1, U.S. Air Force Analysis Agency, 1997.

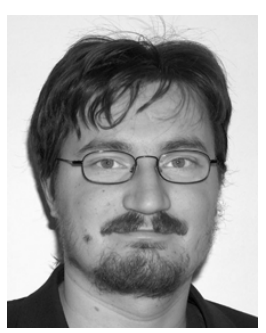

Paul Pop received the Ph.D. in computer science from Linköping University, Sweden, in 2003.

He is a Junior Research Fellow with the Embedded Systems Laboratory, in the Department of Computer and Information Science, Linköping University.

His research interests include design of embedded real-time systems, electronic design automation and hardware/software codesign.

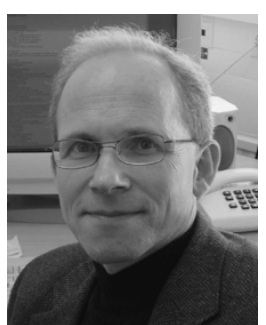

Petru Eles (M'99) received the M.Sc. degree in computer science from the "Politehnica" University of Timisoara, Romania, in 1979, and the Ph.D. degree in computer science from the "Politehnica" University of Bucharest, Romania, in 1993.

$\mathrm{He}$ is currently a Professor with the Department of Computer and Information Science at Linköping University, Sweden. His research interests include design of embedded systems, hardware/software co-design, real-time systems, system specification and testing, CAD for digital systems. He has published extensively in these areas and has coauthored several books including System Synthesis with VHDL (Norwell, MA: Kluwer, 1997) and System-Level Design Techniques for Energy-Efficient Embedded Systems (Norwell, MA: Kluwer, 2004).

Prof. Eles was a corecipient of Best Paper Awards at the 1992 and 1994 European Design Automation Conference (EURO-DAC), and of the Best Presentation Award at the 2003 IEEE/ACM/IFIP International Conference on Hardware/Software Codesign and System Synthesis. He is an Associate Editor of the IEE Proceedings-Computers and Digital Techniques. He has served as a Program Committee Member for numerous International Conferences such as DATE, ICCAD, ECRTS, CASES, EMSOFT, RTSS, and as the Technical Program Chair of the IEEE/ACM/IFIP International Conference on Hardware/Software Codesign and System Synthesis (CODES+ISSS). 


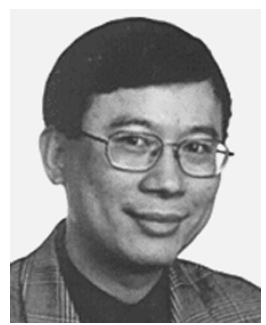

Zebo Peng (M'91-SM'02) received the Ph.D. degree in computer science from Linköping University, Sweden, in 1987.

Currently, he is Professor and Chair in the Department of Computer Systems; he is Director of the Embedded Systems Laboratory; and Chairman of the Division for Software and Systems at Linköping University. His current research interests include design and test of embedded systems, electronic design automation, design for testability, hardware/software codesign, and real-time systems. He has published over 170 journal and conference papers in these areas and is coauthor of System Synthesis with VHDL (Norwell, MA: Kluwer, 1997).

Prof. Peng was corecipient of two Best Paper Awards at the European Design Automation Conferences in 1992 and 1994, and the Best Presentation Award at the 2003 IEEE/ACM/IFIP International Conference on Hardware/Software Codesign and System Synthesis. He has served on the program committees of a dozen technical conferences and workshops, including DATE, DDECS, DFT, ECS, ETW, ITSW, FDL, and MEMOCDE, and was the General Chair of the 6th IEEE European Test Workshop (ETW'01) and the Program Chair of the 7th IEEE Design and Diagnostics of Electronic Circuits \& Systems Workshop (DDECS' 04$)$. He is coeditor of a special issue on Design Methodologies and Tools for Real-Time Embedded Systems in the Journal on Design Automation for Embedded Systems. He is a member of ACM.

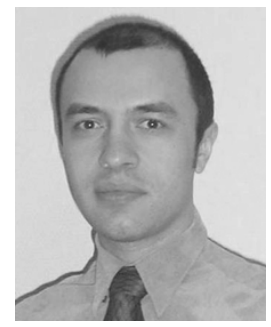

Traian Pop received the M.Sc. degree in software and computer engineering from the Politehnica University of Timisoara, Timisoara, Romania, in 1999, and the Licentiate of Engineering degree from Linköping University, Linköping, Sweden, in 2003, where he is currently pursuing the Ph.D. degree in computer science.

His research interests include real-time systems and design of embedded systems.

Mr. Pop was a corecipient of the Best Presentation Award at the 2003 IEEE/ACM/IFIP International Conference on Hardware/Software Codesign and System Synthesis. 